\title{
Early results from the Whisper instrument on Cluster: an overview
}

\author{
P. M. E. Décréau ${ }^{1}$, P. Fergeau ${ }^{1}$, V. Krasnoselskikh ${ }^{1}$, E. Le Guirriec ${ }^{1}$, M. Lévêque ${ }^{1}$, Ph. Martin ${ }^{1}$, \\ O. Randriamboarison ${ }^{1}$, J. L. Rauch ${ }^{1}$, F. X. Sené ${ }^{1}$, H. C. Séran 1 , J. G. Trotignon ${ }^{1}$, P. Canu ${ }^{2}$, N. Cornilleau ${ }^{2}$, \\ H. de Féraudy ${ }^{2}$, H. Alleyne ${ }^{3}$, K. Yearby ${ }^{3}$, P. B. Mögensen ${ }^{4}$, G. Gustafsson ${ }^{5}$, M. André ${ }^{5}$, D. C. Gurnett ${ }^{6}$, F. Darrouzet ${ }^{7}$, \\ J. Lemaire ${ }^{7}$, C. C. Harvey $^{8}$, P. Travnicek ${ }^{9}$, and Whisper experimenters (Table 1) ${ }^{*}$ \\ ${ }^{1}$ LPCE/CNRS and Université d'Orléans, Orléans, France \\ ${ }^{2}$ CETP/CNRS and VSQP University, Vélizy, France \\ ${ }^{3}$ University of Sheffield, Sheffield, UK \\ ${ }^{4}$ DSRI, Copenhaguen, Denmark \\ ${ }^{5}$ I. R. F. U., Uppsala, Sweden \\ ${ }^{6}$ University of Iowa, Iowa, USA \\ ${ }^{7}$ Institut d'Aéronomie Spatiale de Belgique, Bruxelles, Belgium \\ ${ }^{8} \mathrm{CESR}$, Toulouse, France \\ ${ }^{9}$ Czech Academy of Science, Prague, Czech Republic \\ *The Whisper team deeply regrets the untimely demise of Les Woolliscroft, PI of the DWP instrument and Co-I of Whisper. \\ He played a key role in the Whisper instrument's capabilities.
}

Received: 13 April 2001 - Revised: 13 July 2001 - Accepted: 16 July 2001

\begin{abstract}
The Whisper instrument yields two data sets: (i) the electron density determined via the relaxation sounder, and (ii) the spectrum of natural plasma emissions in the frequency band 2-80 kHz. Both data sets allow for the threedimensional exploration of the magnetosphere by the Cluster mission. The total electron density can be derived unambiguously by the sounder in most magnetospheric regions, provided it is in the range of 0.25 to $80 \mathrm{~cm}^{-3}$. The natural emissions already observed by earlier spacecraft are fairly well measured by the Whisper instrument, thanks to the digital technology which largely overcomes the limited telemetry allocation. The natural emissions are usually related to the plasma frequency, as identified by the sounder, and the combination of an active sounding operation and a passive survey operation provides a time resolution for the total density determination of $2.2 \mathrm{~s}$ in normal telemetry mode and $0.3 \mathrm{~s}$ in burst mode telemetry, respectively. Recorded on board the four spacecraft, the Whisper density data set forms a reference for other techniques measuring the electron population. We give examples of Whisper density data used to derive the vector gradient, and estimate the drift velocity of density structures. Wave observations are also of crucial interest for studying small-scale structures, as demonstrated in an example in the fore-shock region. Early results from the Whisper instrument are very encouraging, and demonstrate that the four-point Cluster measurements indeed bring a unique and completely novel view of the regions explored.
\end{abstract}

Correspondence to: P. Décréau (pdecreau@cnrs-orleans.fr)
Key words. Space plasma physics (instruments and techniques; discontinuities, general or miscellaneous)

\section{Introduction}

After a successful launch in July and August 2000, the four Cluster spacecraft spent about six months in the commissioning phase before starting the formal scientific mission on 1 February 2001. The long duration of the commissioning phase was justified not only by the complexity of the project, but also by the wave instruments which use long double sphere electric antennas. It offered a unique opportunity to make observations at different antenna lengths, and thus, to investigate the wavelength properties of the observed emissions. The antennas were deployed between September and November 2000. Simultaneously, the precession of the Cluster orbit allowed for the exploration of different regions of the magnetosphere. When the WEC (Wave Experiment Consortium) instruments were first powered on, the Cluster orbit was located entirely inside the magnetosphere, with an apogee at about local midnight. The spacecraft encountered the magnetopause boundary for the first time on 17 November 2000 at about 17:00 local time, and entered the solar wind region on 22 December 2000, at about 18:00 local time.

The Whisper (Whisper of HIgh frequency and Sounder for Probing Electron density by Relaxation) experiment is the result of a collaboration between a number of experimenters 
Table 1. The Whisper experimenters

\begin{tabular}{|c|c|}
\hline $\begin{array}{l}\text { P. M. E. Décréau, P. Fergeau, J. L. Fousset, E. Guyot, V. } \\
\text { Krasnoselskikh, L. Launay, M. Lévêque, E. Le Guirriec, Ph. } \\
\text { Martin, O. Randriamboarison, J. L. Rauch, H. C. Séran, } \\
\text { F. X. Sené, J. G. Trotignon, J. P. Villain, C. Vasiljevic }\end{array}$ & $\begin{array}{l}\text { LPCE /CNRS and Orléans University, } \\
\text { Orléans, France }\end{array}$ \\
\hline P. Canu, N. Cornilleau, H. de Féraudy & CETP/CNRS and VSQP, Vélizy, France \\
\hline H. Alleyne, K. Yearby, L. Woolliscroft ${ }^{\dagger}$ & University of Sheffield, Sheffield, UK \\
\hline P. B. Mögensen, M. Jespersen, F. Sedgemore-Schulthess & DSRI, Copenhaguen, Denmark \\
\hline G. Gustafson, M. André & I. R. F. U., Uppsala, Sweden \\
\hline D. A. Gurnett & University of Iowa, Iowa, USA \\
\hline I. Iversen & S. P. W., Lyngby, Denmark \\
\hline J. Lemaire, F. Darrouzet & $\begin{array}{l}\text { Inst. d'Aéronomie Spat. de Belgique, } \\
\text { Bruxelles, Belgium }\end{array}$ \\
\hline C. C. Harvey & CESR, Toulouse, France \\
\hline V. Fiala, P. Travnicek & Czech Acad. Sci., Prague, Czech Republic \\
\hline S. Chapman & University of Warwick, UK \\
\hline
\end{tabular}

(Table 1), organised within the context of the Wave Experiment Consortium, which groups five instruments (Pedersen et al., 1997). The Whisper instrument consists basically of a receiver, a transmitter, and a wave spectrum analyser, completed by the sensors of EFW (Electric Field Wave experiment), and functions of DWP (Digital Wave Processing experiment), which are two other WEC instruments. Whisper is devoted to the survey, both active and passive, of the WEC electric signal in the high frequency range from about 2 to $80 \mathrm{kHz}$, which includes electrostatic and electromagnetic natural emissions of interest to the Cluster objectives. It includes emissions in the vicinity of the plasma frequency, which are essential for density diagnosis, one of the main functions of the Whisper experiment.

The purpose of this paper is to describe the in-flight performance of the Whisper instrument and present examples of data acquired during the early phase of the mission. We emphasise two aspects of the early phase of the Cluster mission: the progressive use of operational capabilities on one hand, and the unveiling of hidden views of the magnetosphere on the other hand. The observations collected so far by the four instruments are so rich that it is impossible to present a complete overview of their content, and many interesting features will not be shown or even mentioned here. Instead we aim to give via a few selected examples, an idea of both the intrinsic performance of Whisper and the characteristics, as revealed by the four-point measurements of some of the magnetospheric structures encountered.

The paper is organised in three main sections. Section 2 illustrates the operation and performance of each individual instrument, in particular, the articulation between its two modes of operation: the sounder, measuring the absolute electron density, and the wave spectrum analyser. Section 3 presents examples of plasma or wave structures observed in different regions of the magnetosphere, and estimations of the drift velocity of density structures. In Sect. 4, we present two examples of four-points observations, and discuss the derivation of the density gradient vector. A brief summary closes the paper.

\section{The Whisper instrument in flight}

\subsection{General design}

The design of the Whisper instrument has benefited from the experience gained in the seventies or eighties (GEOS 1 and 2, ISEE 1, and Viking missions) with magnetospheric sounders, but its technical realisation is rather different. Consequently, its performance is different, and some of the wave features seen in frequency/time spectrograms may look somewhat different.

The design, operational features and performance are described in detail by Décréau et al. (1993, 1997). The electric signals from the four sensors of the EFW instrument (Gustafsson et al., 1997, 2001), i.e. from the high impedance amplifiers located in the vicinity of the spheres, are highpass filtered and fed to the Whisper module where the signals from two opposite sensors enter differential amplifiers and yield effectively one of the two long ( $88 \mathrm{~m}$ tip-to-tip) double sphere dipoles. The Whisper receiver consists of a gain amplifier and filters isolating the $2-80 \mathrm{kHz}$ band, and an 11 bit Analogue to Digital (A/D) converter. The digital signal is used by the Whisper analyser module and controller to generate three products: the number of overflows counted during the acquisition intervals (a few milliseconds every $13.3 \mathrm{~ms}$ ), the total energy of the waveform signal (referred to as the En- 
ergy parameter), and its frequency spectrum as calculated by a FFT processor using frequency bins of $160 \mathrm{~Hz}$ or $320 \mathrm{~Hz}$ resolution in standard modes of operation. Those products, available every $13.3 \mathrm{~ms}$, represent a volume of up to $600 \mathrm{kbits}$ per second. They are compressed on board by the DWP instrument (Woolliscroft et al., 1997) to within the telemetry constraints: $1 \mathrm{kbit}$ per second in normal telemetry mode, and 5 kbits per second in burst mode telemetry. The processing algorithm is chosen by telecommand and thus adapted to the scientific objectives. Typically, 16 samples of each of the three products are accumulated, covering a time interval of about $200 \mathrm{~ms}$, corresponding to a spin angle variation (angular resolution) of about $20^{\circ}$. All accumulated overflow and energy values are transmitted to Earth, as well as a selection of the accumulated spectra (after a quasi - logarithmic compression of the bin amplitudes). The most elaborated product, the spectra, forms the heart of the data set. They are usually presented in form of frequency/time spectrograms, immediately accessible for visual analysis.

The transmitter necessary for the sounder is located inside the Whisper module and connected to the conductive outer braids $2.7 \mathrm{~mm}$ in diameter of one pair of the long EFW antenna, denoted $E_{Y}$ in WEC terminology. The reader can find a schematic diagram of the antenna system used by Whisper in Fig. 1 of Trotignon et al. (2001), and a full description of the sensors and antennas in Gustafsson et al. (2001). The effective length of the transmitting antenna is about $40 \mathrm{~m}$ on each side of the spacecraft. The design of the sounder takes advantage of the possibilities offered by the digital functions present in the Whisper and DWP modules, as described in Sect. 2.2 below.

The Whisper instrument for Cluster 2 is an exact copy of the instrument described by Décréau et al. (1993, 1997), except for two points concerning the receiver: (i) the addition of a switch to allow for the possibility of using either of the two long wire antenna to receive the signal. On Cluster 1, only the $E_{Z}$ (perpendicular to $E_{Y}$ ) antenna was connected to the Whisper receiver; (ii) the suppression of one stage of amplification, leading to possible gains on Cluster 2 of 12 , 24 and $36 \mathrm{~dB}$ instead of $9,21,33$ and $45 \mathrm{~dB}$, as on Cluster 1. When sounding, it is possible to sample the signal stimulated either about $5 \mathrm{~ms}$ after the transmission, as on Cluster 1, or after a longer delay (about $18 \mathrm{~ms}$ ).

After the intense and complete integration campaign for the instruments, their in-flight performance for natural wave measurements was no big surprise. The overall sensitivity (average noise level) is about $210^{-7} \mathrm{~V} \mathrm{~Hz}^{-1 / 2}$. The accumulation of successive Fourier amplitudes reduces the digital and statistical noise until it degrades the sensitivity of the natural mode measurements by only about $2 \mathrm{~dB}$. The dynamic range is determined by the sensitivity of the analogue receiver, the precision of the 11 bit $\mathrm{A} / \mathrm{D}$ converter, and the characteristics of the fast Fourier analyser. It reaches $60 \mathrm{~dB}$ at best for the energy product. It is a window of about $90 \mathrm{~dB}$ for a spectral line, but is limited in each individual spectrum by the flat digital noise present over the complete $80 \mathrm{kHz}$ frequency range. The Blackmann Harris window chosen to reshape the signal before FFT processing carries a calculation noise of about $75 \mathrm{~dB}$ below the level of the highest frequency peak. This specific behaviour has to be taken into account when visually inspecting the frequency/time spectrograms: strong emissions at a given frequency will hide faint features present in other parts of the spectrum which would otherwise be visible. Finally, the frequency and time resolutions reflect the telemetry allocation constraints. The product $\Delta f \times \Delta t$ is about 850 in nominal telemetry mode and 100 in burst telemetry mode.

\subsection{The sounder and the density measurement}

In order to derive the differential values required for multipoint exploration, it is mandatory to make absolute measurements of the total density. In this context, the Whisper sounder provides a reference which can enhance the return of other instruments. The Whisper technique is based on the identification of the electron plasma frequency by analysing the pattern of resonances triggered in the medium by a pulse transmitter. The central frequency of the pulse, a short sinsoidal wave-train, steps in the frequency range of 4-80 kHz (Décréau et al., 1997). Compared to former realisations (Etcheto et al., 1983; Trotignon et al., 1986), the design chosen for Whisper is simpler, short data acquisition after the pulse, leading to a shorter sweep duration. It is also more elaborate; at the end of the sweep, the software reconstructs an active and a passive frequency spectrum by assembling bin packets corresponding to the central frequency of each transmitted wave-train. The "passive" bins are acquired shortly before the wave-train is transmitted, and the "active" bins $13.3 \mathrm{~ms}$ later, shortly after pulse transmission. Each "active" bin which has significantly more signal than the corresponding "passive" bin (about $20 \mathrm{~dB}$ higher), is a potential resonance.

The plasma resonances, characterised by a low group velocity, which can be triggered are:

- the electron plasma frequency, $F_{p}$,

- the electron gyro-frequency $F_{c e}$ and its harmonics,

- the Bernstein waves: upper hybrid frequency and $F_{q}$ resonances.

In a medium with a complex velocity distribution, additional resonances can appear, such as doubling of the $F_{q}$ resonance (Trotignon et al., 2001).

This sounder design has yet to be qualified in space. There were many questions to be answered. Would the duration of the transmitted wave-trains $(0.5 \mathrm{~ms}$ or $1 \mathrm{~ms})$ be sufficient? Would the choice of a short waiting period after the transmission be adequate? How would the pre-amplifier behave in the presence of a large amplitude transmission pulse? Would the three possible transmission levels of 50,100 or $200 \mathrm{~V}$ peak-to-peak be adequate?

A positive answer to these questions came quickly. Clear resonance patterns have been observed even at partial antenna deployment, in the lower altitude part of the orbit (the 


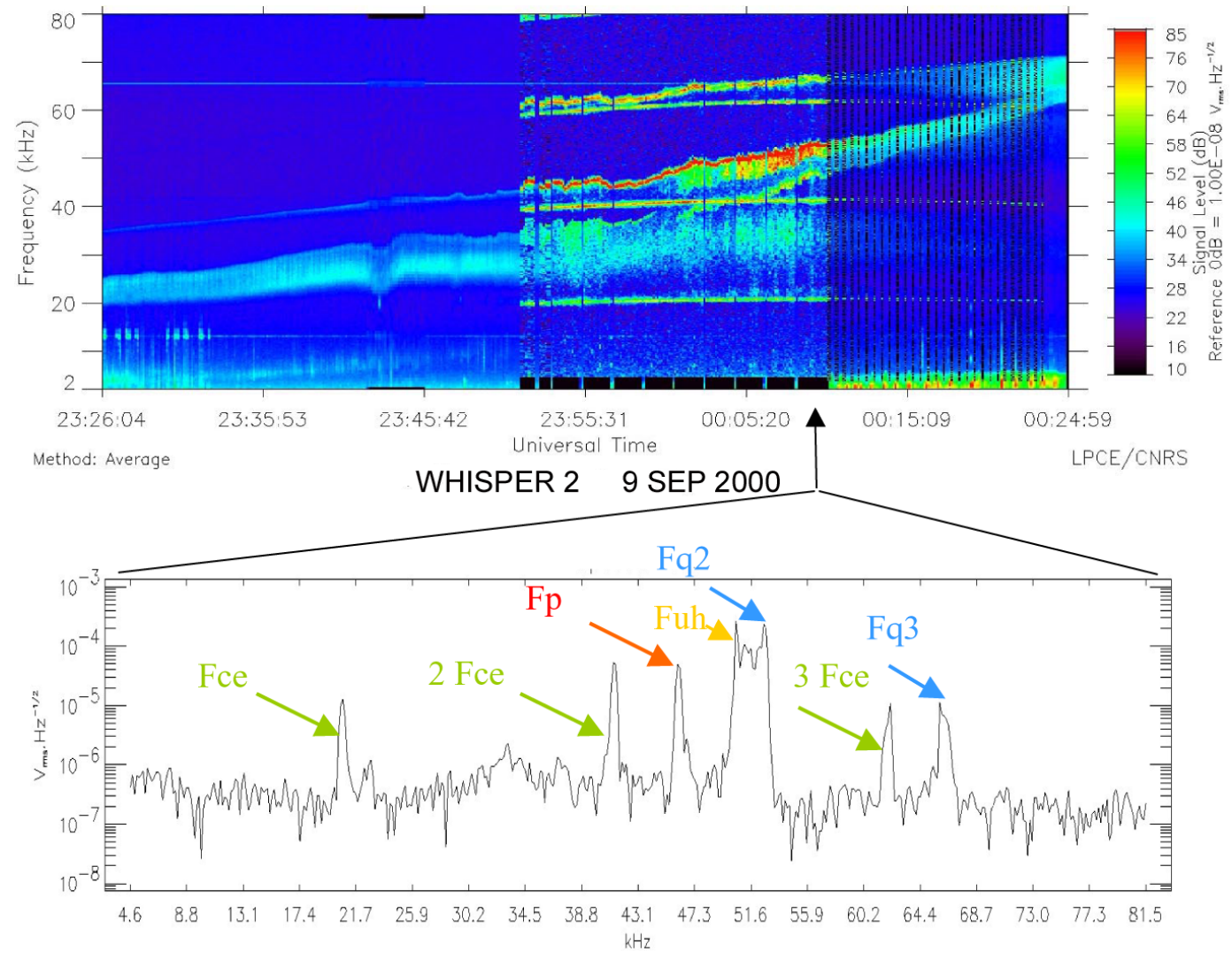

Fig. 1. First demonstration of the capabilities of the sounder in the dayside outer plasmasphere. Upper panel: a frequency/time spectrogram. The operation mode was successively a Natural Wave mode, a Sounding mode, and alternated modes. The bottom panel shows the different resonances observed.

apogee was located in the lobe where the plasma is too tenuous to be measurable by Whisper). Figure 1 shows one of the first observations demonstrating the sounder capabilities. SALSA (SC2) was located in the southern hemisphere, heading toward perigee, at $12 \mathrm{MLT}$, with a $68.5^{\circ}$ invariant latitude and a $4 R_{E}$ geocentric distance in the centre of the time interval. In the first part of the frequency/time spectrogram plotted (before about 23:50 UT), the sounder is not operated. It is almost continuously operated in the second part of the plot. Finally, passive and active operations alternate in the last part, causing vertical stripes in the spectrogram. Active resonant signals are seen for the complete series of gyro-harmonics, Bernstein waves, and the plasma frequency, in comparison to the first and the second parts of the plot; they appear as quasi-monochromatic lines in the single spectrum displayed in the bottom panel.

Three main types of resonance patterns were expected from the empirical knowledge acquired from previous magnetospheric sounders. They have indeed been observed by the Whisper sounder, and they correspond qualitatively to the following plasma regimes:

- a strongly magnetised plasma, where $F_{p}$ lies below a few $F_{c e}$ (Figs. 4 and 5), observed, for instance, in the outer plasmasphere region at low altitude,
- a moderately magnetised plasma, where $F_{c e}$ is still large enough for the different $F_{c e}$ resonances to be distinguished (Fig. 7), and where $F_{p} / F_{c e}$ lies above 3 or 4 , a case which can be observed in the cusp or boundary layer regions,

- a plasma which can be considered as non-magnetised (Figs. 9, 10, and 13), as in the magnetosheath and solar wind regions.

The identification of the plasma resonances, the resonance recognition process, is easy in the last case, since the pattern consists of a single resonance, the plasma frequency $F_{p}$, which is usually easily stimulated. The lowest level of transmission $(50 \mathrm{Vpp})$ is used in non-magnetised regions with good efficiency. The identification is relatively easy for patterns of the second type (Fig. 7). The uncertainty of the frequency position may, however, be higher, as the modulation of resonance amplitudes with antenna attitude relative to the magnetic field direction can reduce the resonance signal. We recommend a medium or high transmission level. In the first case, the resonance recognition is generally relatively easy; when $F_{c e}$ and $F_{p}$ are high enough (as in the case of Fig. 1 and Figs. 4 and 5), a low or medium transmission level can be used in this regime. The resonance stimulation is, however, difficult or even impossible when both $F_{c e}$ and $F_{p}$ are 
low, although the limited duration of the pulse is apparently not a crucial problem; resonances have been stimulated almost down to $4 \mathrm{kHz}$. We have observed cases of low $F_{c e}$ and $F_{p}$ values where the resonance amplitude, even using high transmission levels, is varying along the orbit for reasons not yet fully understood (Debye length is large with respect to antenna length?). Finally, and evidently, no density measurement can be performed in regions where either the plasma frequency is higher than the $80 \mathrm{kHz}$ limit (inner plasmasphere) or the upper hybrid frequency is lower than the $4 \mathrm{kHz}$ limit (lobes and the night sector at a high geocentric distance, in general). Moreover, the level of natural emissions may exceed that of the resonances. High levels of turbulence hiding plasma resonances are, for instance, present on auroral field lines (Fig. 5, first part of the event), or during shock traversals (Fig. 9, around 08:25 and 08:35 UT).

Once the plasma resonance is identified, the density value is directly derived from its frequency position $F_{p}$, according to $N_{e}\left(\mathrm{~cm}^{-3}\right)=F_{p}^{2}(\mathrm{khz}) / 81$. As the sounder instrument suffers only negligibly from the aging that affects other techniques (onboard oscillators offer very high reliability), we can qualify the density measurements obtained from the sounder as absolute measurements.

Concerning natural wave measurements, only in-flight operations could reveal how well or how poorly the instrument's characteristics would fit the fairly large dynamic range covered by the natural emissions of interest in regions that have not been extensively explored. In addition, the capabilities offered by the digital design needed to be tested in space. Specifically, would the directivity measurement be good enough to estimate the wave polarisation? Would the achievable time and frequency resolution be adequate, in particular, to interprete the data set recorded at four different spatial locations?

As soon as the first data was available, at partial antenna deployment (about $36 \mathrm{~m}$ tip-to-tip), the Whisper instrument proved to be well adapted to the survey of natural emissions in the magnetosphere. The sensitivity and dynamic range were close to their predicted values, and no serious fixed-frequency interference was discovered. The sensitivity allows Whisper to measure thermal noise emissions in the Earth's environment, a marginal possibility when using double sphere dipole antennas. The spectrum plotted in Fig. 2 is measured with one of the long electric antenna when fully deployed, with a sphere-to-sphere length of about $88 \mathrm{~m}$. By taking into account the thin wire connecting each sphere to the pre-amplifier located at the end of the boom cable, the minimum measurable electric field level is estimated to be about $2.510^{-9} \mathrm{~V}_{r m s} \mathrm{~m}^{-1} \mathrm{~Hz}^{-1 / 2}$ The spectrum of Fig. 2 was recorded in the magnetosheath, during the long sequence shown in Fig. 9. The level and shape of the thermal noise signal observed above the plasma frequency $F_{p}$ (triggered at $55.4 \mathrm{kHz}$ by the sounder) is of the order of magnitude predicted theoretically (Meyer-Vernet and Perche, 1989) for a simple model of the antenna and an electron temperature of about $50 \mathrm{eV}$. By constructing a more realistic model for the Cluster antenna geometry (Béghin and Kolesnikova, 1998),

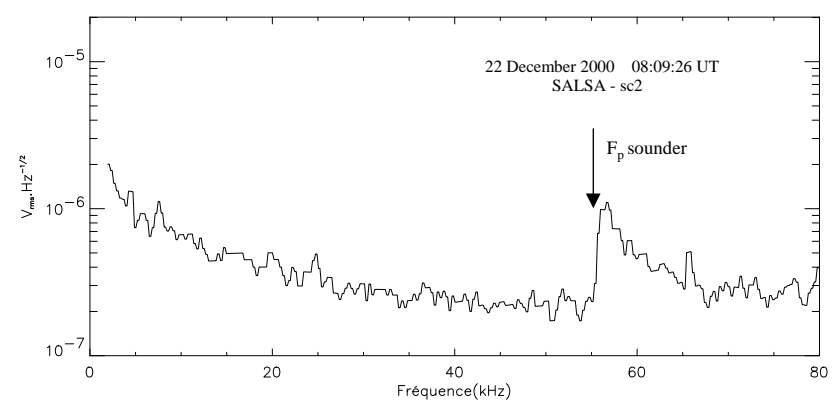

Fig. 2. A single spectrum (16 bin-to-bin accumulations) covering a $213 \mathrm{~ms}$ time interval, taken in the magnetosheath region. The emission above the plasma frequency $(55.4 \mathrm{kHz})$ identified by the sounder, is thought to be electron thermal noise.

we hope to be able to estimate the global electron temperature from the level and shape of the thermal noise signal in the regions free from other natural emissions. In any event, the mere ability to identify the plasma frequency from only a natural emission signature, validated by the sounder, leads to a much better time resolution of the absolute density determination than can be achieved by the sounder alone, and this will be very useful for studying density fluctuations.

\subsection{Natural wave measurements}

Concerning its dynamical range, Whisper behaves as expected. The position of the $90 \mathrm{~dB}$ dynamic range has been adjusted to observations as follows. During early operations, the gain was commuted automatically between the $24 \mathrm{~dB}$ and $36 \mathrm{~dB}$ values, leading to a saturation at a level of $360 \mathrm{mV}_{p p}$, which was reached regularly during AKR emissions. Later on, a 12/24 dB gain commutation cycle was commanded, reducing saturation to a few cases in the solar wind, usually near the shock or in strong AKR source regions. During the science operations phase the gain is regularly commanded to the value predicted in order to give the best compromiseof sensitivity versus saturation for the region being crossed.

The ability to switch from one long double sphere dipole antenna to the other during the commissioning phase allowed for the measurement of the same emissions on antennas of different lengths at close time intervals, or at close distance. An example of similar signals measured at three antenna lengths is shown in Fig. 3. SAMBA (SC3) antennas are deployed, respectively, at $20 \mathrm{~m}$ (46 m sphere-to-sphere) for the $E_{Z}$ double sphere dipole, and at $41 \mathrm{~m}(88 \mathrm{~m}$ sphereto-sphere) for the $E_{Y}$ dipole, while TANGO (SC4) antennas were deployed at $36 \mathrm{~m}$ sphere to sphere, both for $E_{Z}$ and $E_{Y}$ dipoles. The upper and middle panels display the frequency/time spectrograms observed in the auroral region, evening sector (GSE coordinates at 1.5, 2.0 and $-5.7 R_{E}$ ), respectively, on SAMBA (top) and TANGO, located at approximately a $420 \mathrm{~km}$ separation. At the start of the sequence, AKR emissions are observed in the upper part of the frequency range, giving way at about 11:45 UT to a type III solar burst event. Hiss emissions are constantly present 

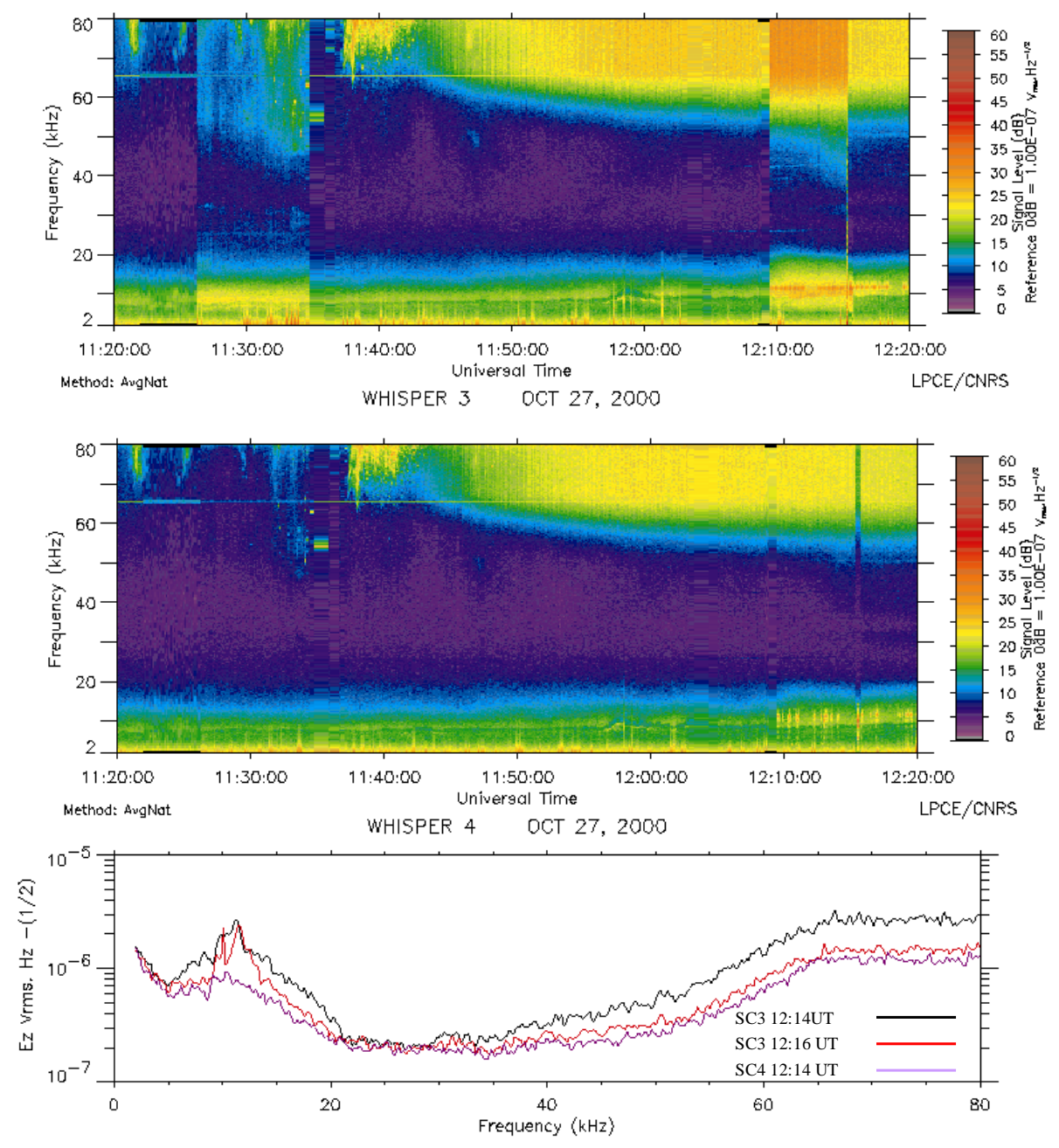

Fig. 3. Comparison of the potential difference measured on double sphere dipoles at three different sphere-to-sphere lengths: $46 \mathrm{~m}$ (most of the time) and $88 \mathrm{~m}$ (a few minutes centered at 11:30 and 12:12 UT) on SAMBA (SC3, upper panel); $36 \mathrm{~m}$ on TANGO (SC4, middle panel). The bottom panel displays single spectra measured at nearly the same time and place. The potential differences measured are proportional to the dipole length for the type III solar burst emissions (after 11:40 UT, above $40 \mathrm{kHz}$ ).

in the low frequency range. The Whisper differential receiver is connected on both spacecraft to the $E_{Z}$ dipole, except for a few minutes around 11:30 and 12:12 UT, when both SAMBA and TANGO receivers are switched to the $E_{Y}$ dipole. The signal intensity displayed in the spectrograms is the potential difference measured between the spheres in physical units, taking into account the EFW and Whisper transfer functions. The transition when switching antennas is quite clear in the upper plot, as the sensitivity is almost doubled when the dipole length goes from 46 to $88 \mathrm{~m}$, whereas it does not change on TANGO (bottom). The lower panel of Fig. 3 presents a detailed comparison of single frequency spectra obtained during the second antenna transition: two successive spectra on SAMBA, one on TANGO. The electric field intensity of type III solar burst emissions is expected to be the same at the two spacecraft locations, since the sources are very distant over the short time period considered, as they appear to be stationary (TANGO observations). The electronic noise level, apparent at about $25 \mathrm{kHz}$ (same level on all three spectra, measured also on SALSA, as shown in Fig. 2) can be subtracted from the different signals. The resulting levels $\left(1.1,1.4\right.$ and $\left.2.7 \mu \mathrm{V}_{r m s} \mathrm{~Hz}^{-1 / 2}\right)$ are almost in perfect proportion to the respective lengths of the antennas $(36,46$ and $88 \mathrm{~m}$ ). We can conclude from this particular study that the potential difference versus electric field ratio is actually close to the full dipole length, at least when the wave length of the corresponding emissions is sufficiently large.

Finally, it has been proven that Whisper can detect a spin modulation at different frequencies of a spectrum during the same time interval. As explained above, the analyser calculates frequency spectra of the electric field power in the 2$80 \mathrm{kHz}$ range every $13.3 \mathrm{~ms}$ and before transmission to Earth, accumulates them for a time period significantly shorter than the spin duration. The standard accumulation duration (about $200 \mathrm{~ms}$ ) corresponds to the spacecraft rotation of about $20^{\circ}$, but that value can be lowered for specific operations. In the burst mode telemetry rate, it is possible to transmit several of the standard accumulated spectra during a spin period of $4 \mathrm{~s}$, 

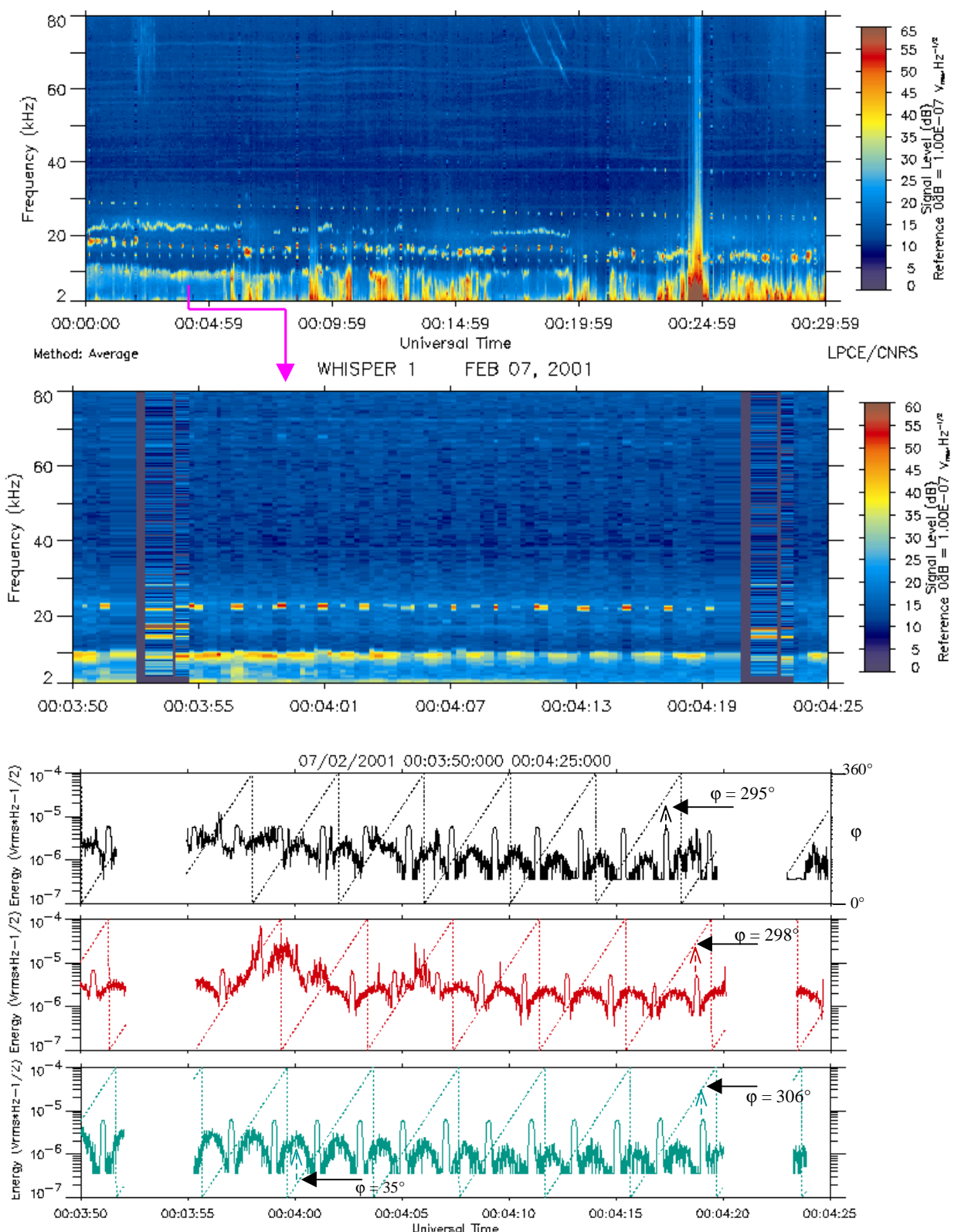

Fig. 4. Frequency/time spectrograms measured on RUMBA (SC1) in the auroral region (two upper panels), and energy and phase angle versus time from spacecraft 1 to 3 (lower panels). The phase angle variations (dotted lines) indicate similar spin periods (from 4.003 to $4.017 \mathrm{~s}$ ). The energy parameter (solid line) is the total energy (in units of potential difference measured by the $88 \mathrm{~m}$ dipole) measured in the $2-80 \mathrm{kHz}$ band. Emissions at $F_{p}$ (about $10 \mathrm{kHz}$ ), display a spin modulation peaking along the magnetic field direction. Emissions at $F_{X}$ (about $22 \mathrm{kHz}$ ) display unusual spin modulation features present on all four spacecraft (standard Cluster color codes: black, red, green, respectively for spacecraft $1,2,3$ ). Spacecraft 4 data, not shown, displays similar features.

with twelve of them in the WEC basic operations. Moreover, the energy parameter product, which can be transmitted at a significantly higher time resolution $(13.3 \mathrm{~ms})$ completes the picture.

The event presented in Fig. 4, taken in the night side auroral region ( $75^{\circ}$ invariant latitude, $02 \mathrm{MLT}, 4.9 R_{E}$ geocentric distance), has been chosen to clearly illustrate Whisper's ability to depict spin modulations. In this particular case, the Whisper frequency/time spectrogram from the RUMBA spacecraft ( $\mathrm{SC1}$ ) is presented in the upper panel over a 30 min time interval. This plot shows a narrow band emission at about $22 \mathrm{kHz}$, a frequency called $F_{X}$ hereafter, located between the first and the second harmonic of the electron gyrofrequency $\left(F_{c e} \approx 14.5 \mathrm{kHz}\right)$. The emission varies slowly but surely with time, in both frequency and amplitude. The electron gyro-frequencies not associated with any signature in the natural emissions are revealed by the sounder, which operates every $28 \mathrm{~s}$ (see Trotignon et al., 2001). The upper hybrid frequency $F_{u h}$, observed in the $15-20 \mathrm{kHz}$ band, is always stimulated by the sounder, associated at times with 


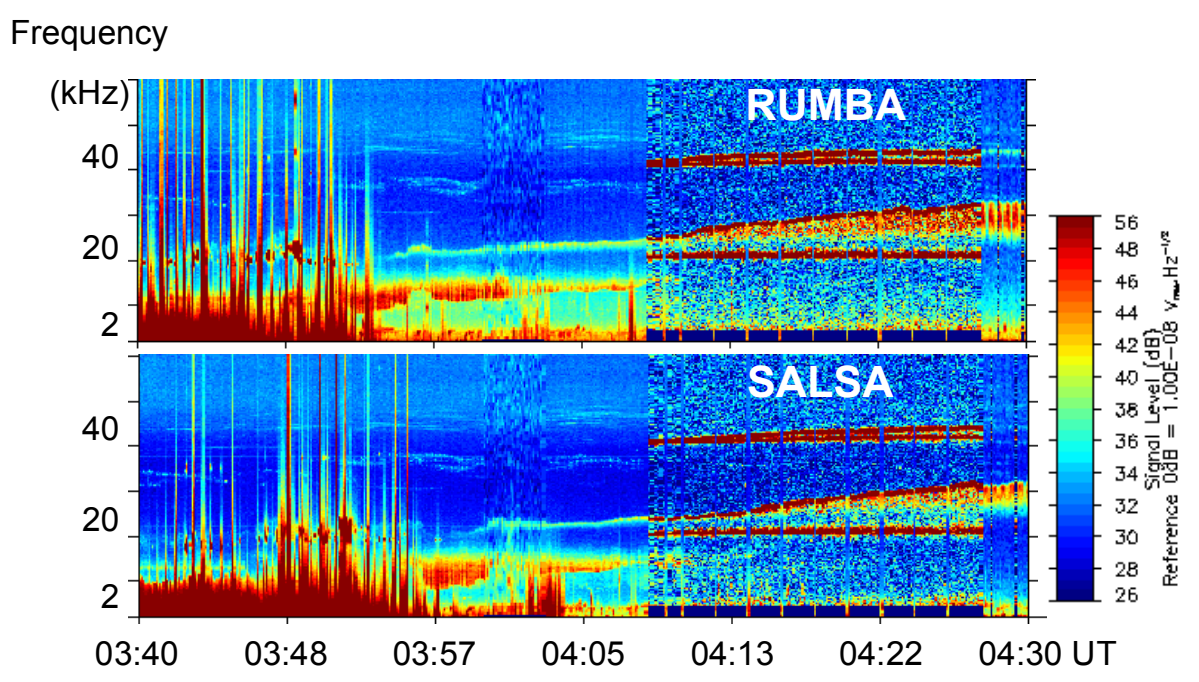

Fig. 5. Comparison of RUMBA and SALSA frequency/time spectrograms, illustrating several properties of the wave emissions in the internal magnetosphere on 6 October 2000 (9.5 MLT, $-75^{\circ}$ invariant latitude, $4.3 R_{E}$ ).

strong natural emissions. The middle and lower (three) panels of Fig. 4 represent detailed observations during a $35 \mathrm{~s} \mathrm{time}$ interval, including two sounding operations. In the middle panel, the sounding operations can be recognised by the limitation of the individual active spectra to a lowest frequency of $4 \mathrm{kHz}$. The three bottom panels display the energy and the phase angle parameters respectively, for spacecraft 1 (black curves), spacecraft 2 (red curves) and spacecraft 3 (green curves), with the periods of sounding operations left blank since the energy parameter is not available at that time. Incidentally, this example illustrates the good synchronisation of the operations on the four spacecraft. spacecraft 4 observations, not shown here in order to maintain readability, are similar to those of the other Cluster spacecraft.

A striking feature in the detailed plots in Fig. 4 is the fact that the $F_{X}$ emission at about $22 \mathrm{kHz}$ is primarily appearing only at a very specific angle, a few degrees wide in the spin phase at a quarter of a spin of the overall modulation in energy. The overall modulation is associated with the emission at about $10 \mathrm{kHz}$ which marks the upper range of the hiss emissions that are present over almost the entire 30 min duration of the event, as shown in the upper panel. After a study of the sounder's signatures during the complete event, we could conclude that the emission around $10 \mathrm{kHz}$ corresponds to the plasma frequency, as the observed pattern matches exactly the relationship $F_{u h}^{2}=F_{p}^{2}+F_{c e}^{2}$, where $F_{c e}$ is the recognised electron gyro-frequency, and $F_{u h}$ (the upper hybrid frequency) is the main resonance triggered by the sounder between $F_{c e}$ and $2 F_{c e}$, varying in frequency during the event.

The overall modulation of the energy parameter (the secondary wide peak), due to the modulation at $F_{p}$, resembles a sinusoidal modulation at twice the spin frequency, as expected for a polarised wave. In order to determine the direction of the receiving antenna with respect to the magnetic field when the energy at $F_{p}$ is maximal, we use the spacecraft 3 data (green curves), which are particularly clean around 00:04 UT. The phase angle of maximum $F_{p}$ energy is about $35^{\circ}$. Assuming that the spacecraft spin axis is exactly along the GSE $Z$-axis (the directions differ by only a few degrees) and a phase angle of $35^{\circ}$ is present, this means that the $Y_{B}$ spacecraft body build axis, located in the spin plane, has rotated through $35^{\circ}$ since the time it was in the GSE $X Z$ plane (the origin of phase angle). It is thus pointing toward the morning sector (keeping in mind that the spacecraft rotation vector is opposite to that of the Earth). The $E_{Z}$ receiving antenna is at $45^{\circ}$ to $Y_{B}$. In particular, the WEC2 sensor $\left(-E_{Z}\right)$ is located in the spin plane at a phase angle of $10^{\circ}$ when $Y_{B}$ is at $+35^{\circ}$. The DC magnetic field (GSE components 441,92 and $225 \mathrm{nT}$ ), projected into the spin plane, corresponds to a phase angle of $12^{\circ}$. The signal measured at $F_{p}$ is consequently found to be maximal when the receiving antenna is (within the few degrees of uncertainty of the phase estimation) at its closest alignment with the magnetic field direction. Such behaviour is not surprising from a longitudinal Langmuir wave (see, for instance, Thiel and Debrie, 1981, for estimations of signal levels received at different angles from a local dipolar source).

The modulation associated with $F_{X}$ is of a different nature. The peculiar, unexpected feature (a narrow peak) of the spin modulation seems to indicate some kind of interference, whereas time variations of the frequency and amplitude favour interpretation in terms of a natural phenomenon. Such an interference, if it exists, is not due to another active scientific instrument; EDI is off on spacecraft 4, as is ASPOC on spacecraft 1. It does not seem to be related to the EFW sensors, as high resolution EFW products (DC potential differences) are clean.

The observations from the four spacecraft show similar behaviour concerning the phase angle of maximal $F_{X}$ emission around 00:04 UT: $295^{\circ}, 298^{\circ}, 306^{\circ}$ and $285^{\circ}$ for spacecraft 1 to spacecraft 4 , respectively, corresponding to phase posi- 
tions of the receiving antennas $\left(250^{\circ}, 253^{\circ}, 261^{\circ}\right.$ and $\left.240^{\circ}\right)$ close to perpendicular to the magnetic field direction. However, this fact is purely accidental, as $\mathbf{3 5} \mathrm{min}$ later, the phase angle of the maximal $F_{X}$ emission corresponds to an angle of about $27^{\circ}$ between $E_{Z}$ and the magnetic field direction projected onto the spin plane. On the other hand, the receiving antenna attitude at the $F_{X}$ emission is neither aligned with the Sun's direction, nor with a crude estimation of the plasma drift velocity derived from EFW electric field measurements. Although we are still far from an interpretation of this unusual event, we would like to point out that the Whisper observational capabilities are at least providing interesting clues, which would have been hidden to the former SFA type of wave instruments.

\section{Plasma and wave structures}

\subsection{Internal magnetosphere}

The frequency/time spectrograms obtained from the four Whisper instruments are usually very rich in information, and a simple visual inspection reveals much about the regions crossed. They inform us about two key parameters, the magnetic field strength and the electron density, as seen in the plasma resonance pattern triggered during the sounder operation (Trotignon et al., 2001). The natural wave emissions bring other important clues about the region. They not only inform us about local plasma conditions, such as the turbulence level in the higher frequency range or the propagation characteristics (Canu et al., 2001), but also provide a view from a distance of the surrounding region. In some cases, Whisper detects local signatures from transported populations, informing us about the proximity of their source (an example is shown in Sect. 4.1). In other cases, the wave features observed are due to distant electromagnetic sources, sometimes internal to the magnetosphere, such as a nonthermal continuum or AKR, and sometimes really remote, such as type III solar bursts. The nature of the wave can be characterised by a comparison of its signatures viewed from the different spacecraft; for this, two spacecraft are generally sufficient. An example of wave emissions in the magnetosphere is shown in Fig. 5. The spacecraft, located over the southern hemisphere, are travelling inbound from an auroral region to the plasmatrough (see Fig. 6, bottom). In the centre of the time interval (04:05 UT), they are located at $75^{\circ}$ invariant latitude, 9.5 MLT and a $4.3 R_{E}$ geocentric distance. The instrument is operated in a Natural Wave mode during the first part of the sequence, then from about 04:13 UT it is operated, primarily in Sounding mode (a sounder operation can be recognised by the position at $4 \mathrm{kHz}$ of the low frequency boundary).

The electron gyro-frequency, around at $20 \mathrm{kHz}$, indicating a magnetic field amplitude of about $700 \mathrm{nT}$, is detected from the resonance pattern triggered by the sounder. The second harmonic is sitting at about $40 \mathrm{kHz}$. The upper hybrid frequency is observed to increase in time from about 20
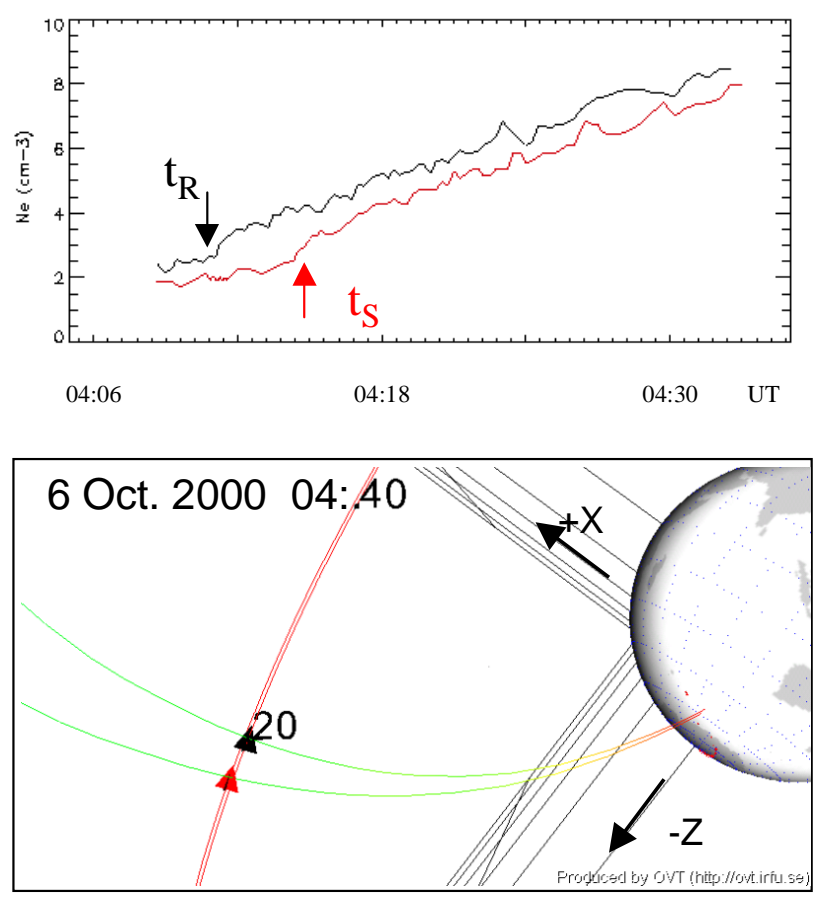

Fig. 6. Upper panel: density profiles (black for RUMBA, red for SALSA) for a time interval shown in Fig. 5. Lower panel: configuration of the 2 spacecraft trajectories (red) and magnetic field lines (green) plotted by the OVT visualization tool. Triangles (standard Cluster color-code) show the spacecraft positions at 04:20 UT. The orbit trajectory is almost perpendicular to the field lines, along which we think the density structure is aligned.

to $30 \mathrm{kHz}$, leading to the density variation shown in Fig. 6 (top). These densities are characteristic of magnetic field tubes located on the day side that are in the process of being replenished from the diurnal ionospheric source (Décréau et al., 1982, Carpenter et al., 1993).

Three types of waves, observed as three different layers, seem to coexist, as is confirmed by two spacecraft observations:

- Observed exactly at the same time on both spacecraft, light blue structured elements are visible above $30 \mathrm{kHz}$. They are electromagnetic emissions, probably propagating non-thermal continuum radiation (Gurnett, 1975; Etcheto et al., 1982). These emissions, homogeneous in space, are variable with time.

- Wave signatures related to a local density structure, which is apparently stationary with time, but clearly not in space. One signature is the emission at the upper plasma frequency. The strong resonance observed after 04:08 UT, between 20 and $30 \mathrm{kHz}$ in the $F_{c e}-2 F_{c e}$ branch, is interpreted as $F_{u h}$. It is clearly linked to a natural emission feature present before sounding. A second signature, better seen on RUMBA in the central part of the plot, is the lower cutoff at about 8 to $12 \mathrm{kHz}$ of the emissions displayed in the orange colour code. Such "trapped continuum radiation" events have been 


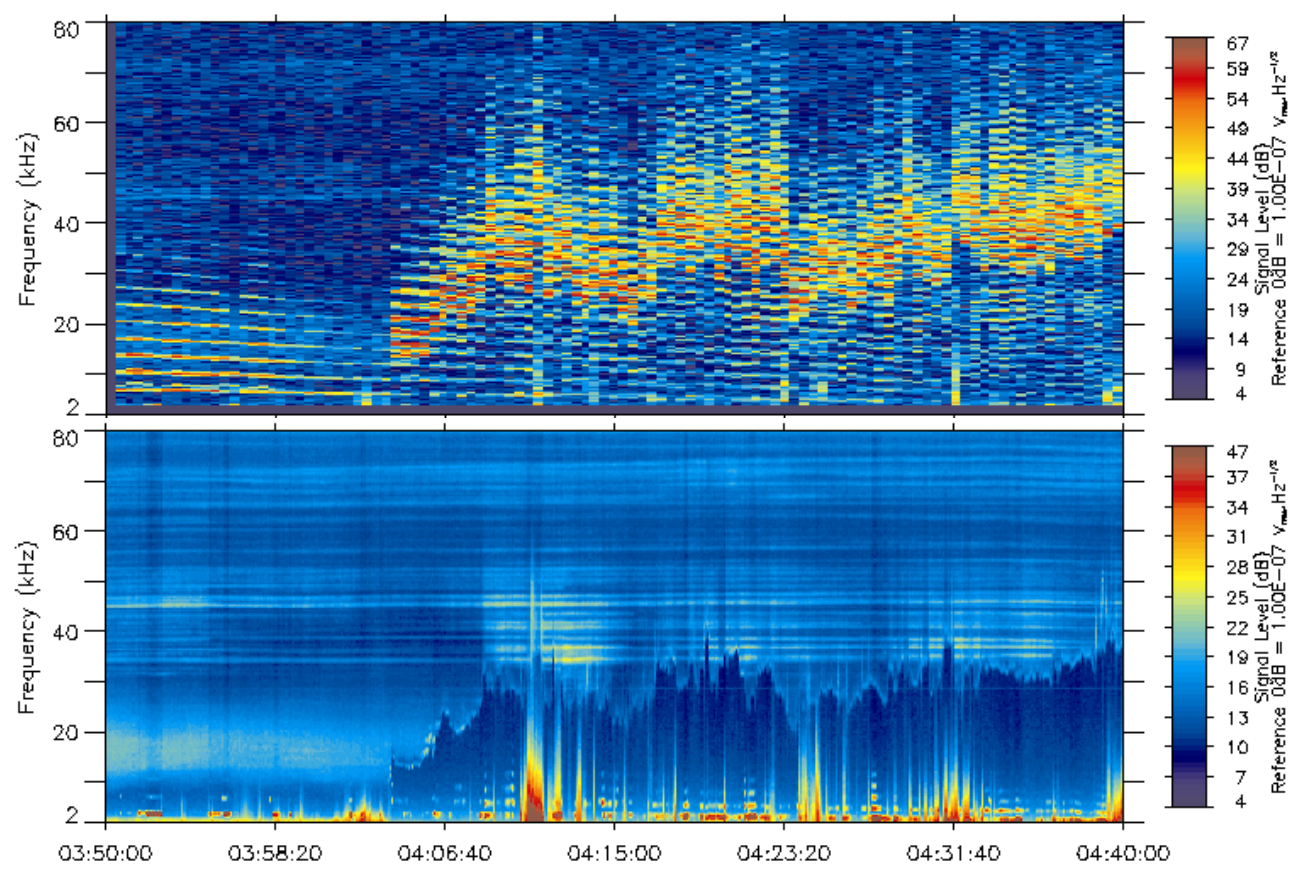

Fig. 7. Frequency/time spectrogram in Sounding only (upper panel) and Natural wave only (bottom panel) modes, derived from TANGO (spacecraft 4) data in the dayside region ( $11.7 \mathrm{MLT}, 81^{\circ}$ invariant latitude, $8.1 R_{E}$ ), outbound leg, on 26 Feb 2001 . The sharp density increase at about 04:06 UT marks the entry in a plasma cloud, left at 05:00 UT, inside the magnetosphere. The magnetopause proper is crossed at 06:10 UT.

observed on GEOS or ISEE (Etcheto et al., 1982). Their lower cutoff frequency is the plasma frequency $F_{p}$, as indicated by the $F_{c e}, F_{u h}, F_{p}$ relationship. Both signatures are related to plasma density variations which are observed first on RUMBA, then on SALSA, but which look similar on the two satellites.

- During the first part of the pass, in a dark red colour at the lowest frequencies of the spectrogram, structures are stable neither in time nor in space (no clear correlation can be distinguished between RUMBA and SALSA observations). They are intense bursty emissions of a short duration, interpreted as electrostatic emissions linked to wave-particle interactions, known to be active in the auroral region (Dubouloz et al., 1991).

The density profiles plotted in the upper panel of Fig. 6 are similar, with a time delay between SALSA and RUMBA, $t_{S}-t_{R} \cong 205 \mathrm{~s}$, which is almost constant during $20 \mathrm{~min}$ or more. The configuration of the spacecraft orbit and magnetic field line, derived by the Cluster Orbit Visualisation Tool (OVT: see Stasiewicz, 2001) is shown in the lower panel of Fig. 6. The Cluster orbit (red lines) for RUMBA (black symbol) and SALSA (red symbol) are almost perpendicular to the magnetic field lines (green lines), along which we think the density irregularity is aligned. On the other hand, the angle between the orbital velocity vector $\boldsymbol{V}$ of the constellation and the line joining the two satellites is quite low $\left(8^{\circ}\right)$. Projected along the velocity vector, the spacecraft separation is $1165 \mathrm{~km}$, which when combined with the time delay of
$t_{S}-t_{R}$, leads to a relative velocity of $5.65 \mathrm{~km} \mathrm{~s}^{-1}$ between the spacecraft pair and the density structure. This is $10 \%$ higher than the spacecraft orbital velocity, implying that the quasi-stationary structure is probably travelling away from the Earth at about $0.5 \mathrm{~km} \mathrm{~s}^{-1}$. The wealth of density data obtained from the four spacecraft since the start of the scientific phase of the mission is expected to provide better and more complete information about the dynamics of plasma structures in the outer plasmasphere region.

\subsection{Plasma structures in the noon sector region}

In the noon sector, when travelling outbound from the internal magnetosphere towards the magnetosheath, Cluster often encounters magnetic field tubes loaded with a significant plasma density. The origin of the large plasma bubbles observed by Whisper in this region is not obvious. Are the spacecraft crossing a detached plasmaspheric region? Are they actually crossing the cusp proper, or simply approaching this region, in which so many field tubes are converging?

One such event is shown in Fig. 7 from an outbound pass in the northern hemisphere. At 04:15 UT, the position is about $11.7 \mathrm{MLT}, 81^{\circ}$ invariant latitude, at a $8.1 R_{E}$ geocentric distance (close to the expected location of the cusp). The top panel groups the spectra obtained in Sounding mode, operating about twice a minute. The bottom panel groups the spectra obtained in Natural mode at a recurrence of about 3 per second. Here, the telemetry rate is in burst mode, which permits the highest instrument performance. 


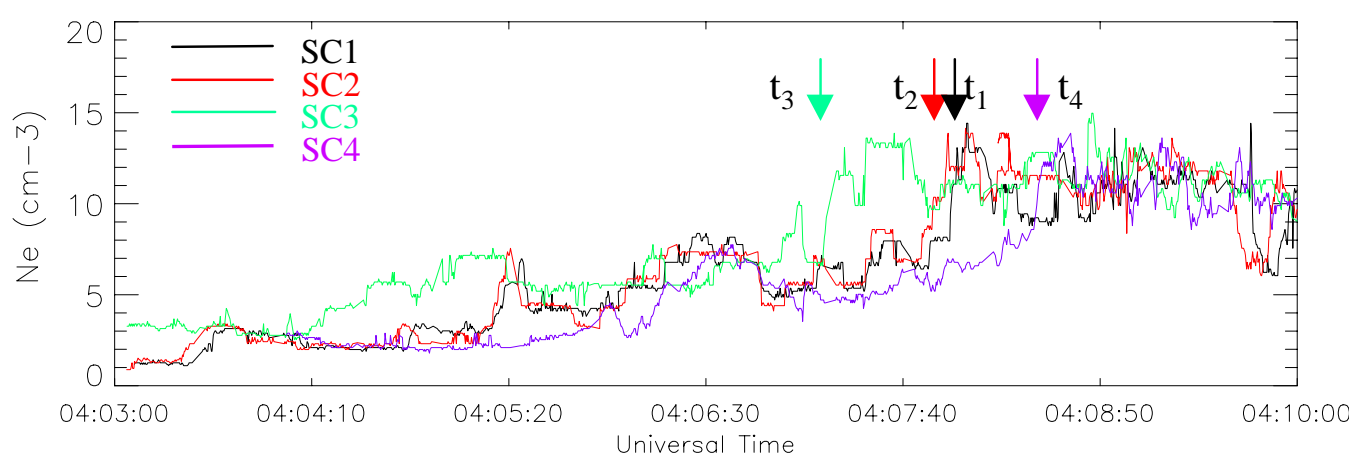

Fig. 8. Comparison of the density profiles for a short, $7 \mathrm{~min}$ time interval during the event shown in Fig. 7 . The arrows indicate the occurrence times of the main density gradient, as observed on each satellite.

The magnetic field amplitude is slowly decreasing, as indicated by the frequencies of the harmonics of the electron gyro-frequency, visible in the top panel $\left(F_{c e} \approx 3 \mathrm{kHz}\right.$ around 04:05 UT, in agreement with magnetic field measurements of about 100 nT). In this panel, one can observe after 04:05 UT, in addition to the gyro-frequency resonance pattern, a wide band of strong resonances (appearing in red around $30 \mathrm{kHz}$ ). They are located at the plasma frequency $F_{p}$ and above. The density increased suddenly at 04:03 UT ( $F_{p}$ climbing from below 4 to above $30 \mathrm{kHz}$ ). Its variations display a wavy structure which is seen in more detail in the bottom panel. Actually, the bottom panel shows an example of how well the plasma frequency is defined by the low frequency cutoff of background electromagnetic radiation. In this particular case, the non-thermal continuum radiation above $30 \mathrm{kHz}$ exhibits a series of narrow band elements, very stationary in time, but modulated in amplitude (see Fig. 3 in Etcheto et al., 1982, showing a similar looking frequency/time spectrogram obtained on ISEE, close to the equator, at 7.9 MLT and about $\left.9.2 R_{E}\right)$.

The variations with time of the density are different on each spacecraft, as illustrated in Fig. 8, which shows a few minutes at the start of the density increase. A sharp gradient, present in each of the four density profiles, marks the entry into the high density part of the bubble. It occurs at times $t_{1}$ to $t_{4}$, as indicated in the figure. From the times $t_{i}$ (known with precision $\pm 1 \mathrm{~s}$ ) and corresponding positions $\boldsymbol{r}_{i}$ of the four spacecraft at the discontinuity crossings (uncertainty in the position given by the Cluster operation centre), it is possible to determine the velocity $\boldsymbol{V}$ of the discontinuity, assuming a rigid plane travelling at a constant velocity along its normal $\boldsymbol{n}$, thus satisfying: $\left(\boldsymbol{r}_{i}-\boldsymbol{r}_{j}\right) \cdot \boldsymbol{n}=V\left(t_{i}-t_{j}\right)$, where $\boldsymbol{V}=V \boldsymbol{n}$ (Chanteur, 1998). This leads to the following $\boldsymbol{V}$ components in the GSE frame: $-5.9,0.0,1.9 \mathrm{~km} \mathrm{~s}^{-1}$, with an uncertainty in magnitude of $10 \%$, and an uncertainty in direction of a few degrees. The plasma bubble is travelling toward the Earth with a velocity of about $6.2 \mathrm{~km} \mathrm{~s}^{-1}$, almost opposite (within $20^{\circ}$ ) that of the orbital velocity, and almost perpendicular (at an angle of about $85^{\circ}$ ) to the DC magnetic field.

If the structure crossed is a 1-D structure, then since the spacecraft are travelling at similar orbital velocities, they should see the same time profile for the density. Actually, by applying the appropriate time translation, we obtain a good superposition of all profiles within a time interval of about a minute. Outside the interval, the assumption of a rigid, 1-D, structure travelling at constant velocity fails. Nevertheless, such an analysis provides an interesting clue about the nature of the plasma bubble. Since the electron gyro-frequencies can be distinguished on the frequency/time spectrograms, we think that the bubble is not related to the exterior cusp, but rather to magnetic field tubes connected to the magnetopause boundary layer. The original plasma source could be the magnetosheath or the plasmasphere. In any event, the anti-sunward convection of a structure quasi-parallel to the magnetic field is clear in this case. In order to understand properly the shape and dynamics of the structures observed, a more detailed study than can be presented here is needed. Detailed studies are indeed to be conducted in this key interface region, where other events of the same type, i.e. with similar large irregularities in density, have been observed.

\section{Four-points Whisper observations}

\subsection{The bow shock and foreshock region}

Impressive views of the shock and foreshock regions have been obtained in late December 2000, when the Cluster orbit was skimming the bow shock surface in the 17 MLT sector. Figure 9 shows the first encounter of the bow shock on 22 December 2000 close to the apogee. The frequency/time spectrograms of the four spacecraft are displayed, as well as the density variations obtained from the plasma frequency signatures. SALSA (spacecraft 2) clearly stayed longer in the solar wind during this first incursion around 08:30 UT, which lasts about $12 \mathrm{~min}$. It has been shown that the solar wind is indeed pushing the magnetosphere towards the Earth and that SALSA, at that point is located westward of the other spacecraft, is the first to be swept by the boundary when it moved toward the Earth, and the last when it receded.

One beautiful example of foreshock wave emissions is shown in Fig. 10, taken from SAMBA (spacecraft 3), over about a $30 \mathrm{~min}$ time interval. The Cluster constellation had 


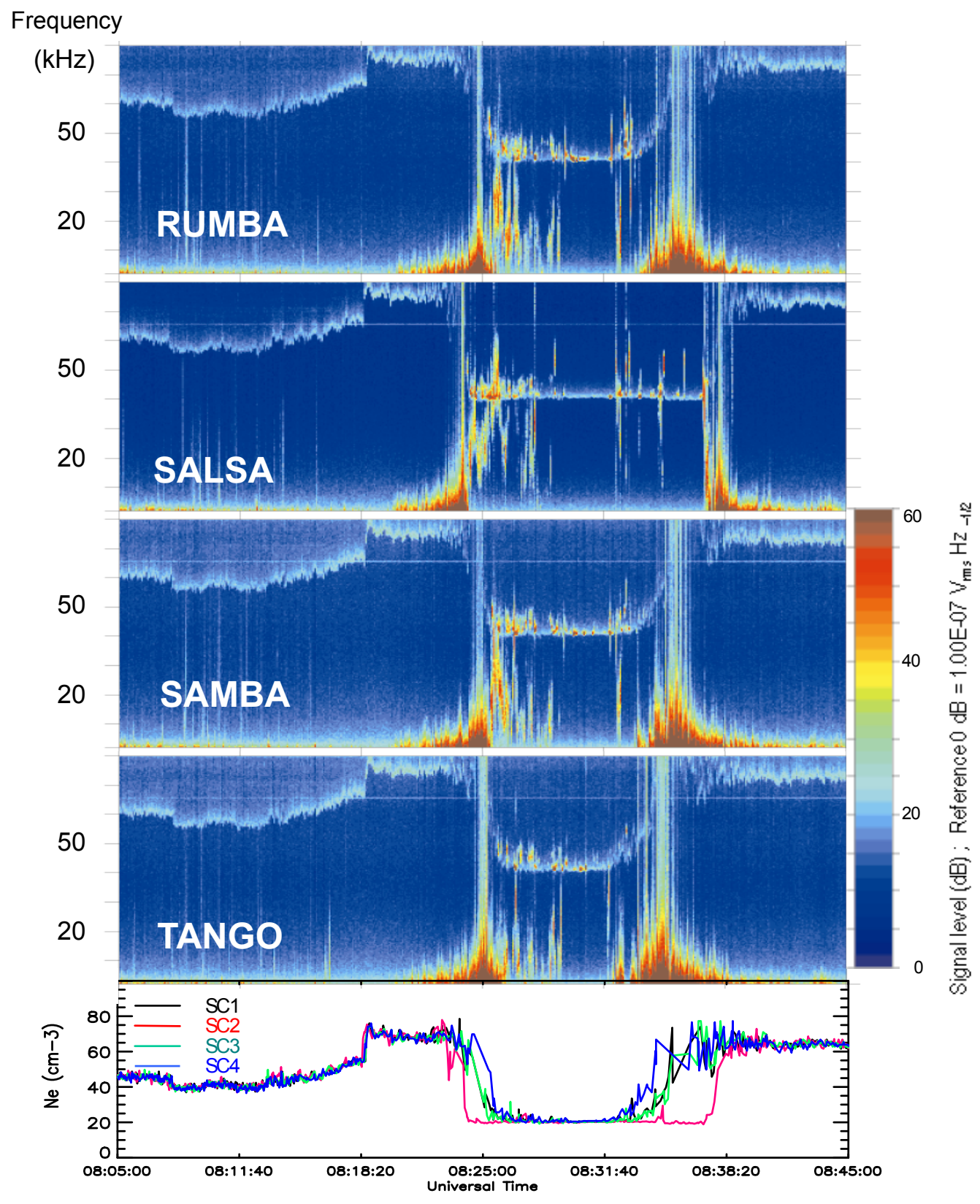

Fig. 9. Frequency/time spectrograms of the four spacecraft during a bow shock crossing (22 December 2000), and the corresponding density profiles (bottom panel). The RUMBA (spacecraft 1), SALSA (spacecraft 2), SAMBA (spacecraft 3) and TANGO (spacecraft 4) spacecraft are located at 17:00 LT, close to apogee $\left(12^{\circ}\right.$ latitude, $\left.19.5 R_{E}\right)$.

crossed the quasi-perpendicular shock region (as depicted from the DC magnetic field configuration), from downstream to upstream, and entered the electron foreshock. All Cluster spacecraft display similar, but not identical, festoon-like features, during about four hours. Such bursty oscillations around the plasma frequency have been reported by many authors (Anderson et al., 1981; Etcheto and Faucheux, 1984; Fuselier et al., 1985). A characteristic feature of these oscillations is their intermittent character and their rather large local frequency band. Another feature which has given rise to much discussion is the position of their characteristic fre- quency, which is sometimes well below the local plasma frequency. It was found that very near to the electron foreshock boundary (defined by fast electrons streaming along magnetic field lines tangent to the bow shock), the electric field spectrum occurs in a narrow frequency band centred around the electron plasma frequency; deeper in the foreshock region, on shock-connected field lines, the spectrum spreads both upward and downward in frequency (Etcheto and Faucheux, 1984; Fuselier et al., 1985). Whisper, with its specific capabilities, provides a new view of these oscillations. In Fig. 10, the upper panel presents the Whisper fre- 

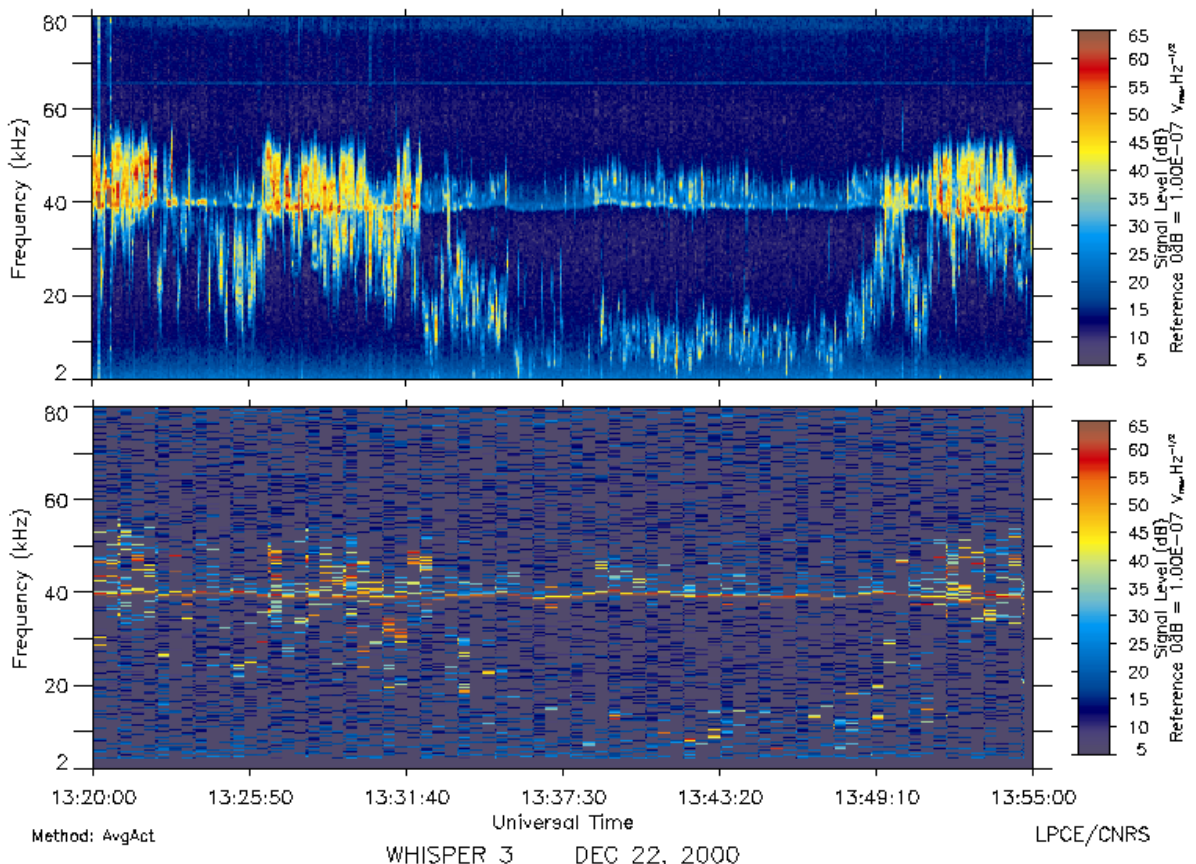

Fig. 10. Frequency/time spectrogram in Natural wave mode only (upper panel) and in Sounding mode only (lower panel) measured by SAMBA (spacecraft 3) in the foreshock region (22 December 2000).

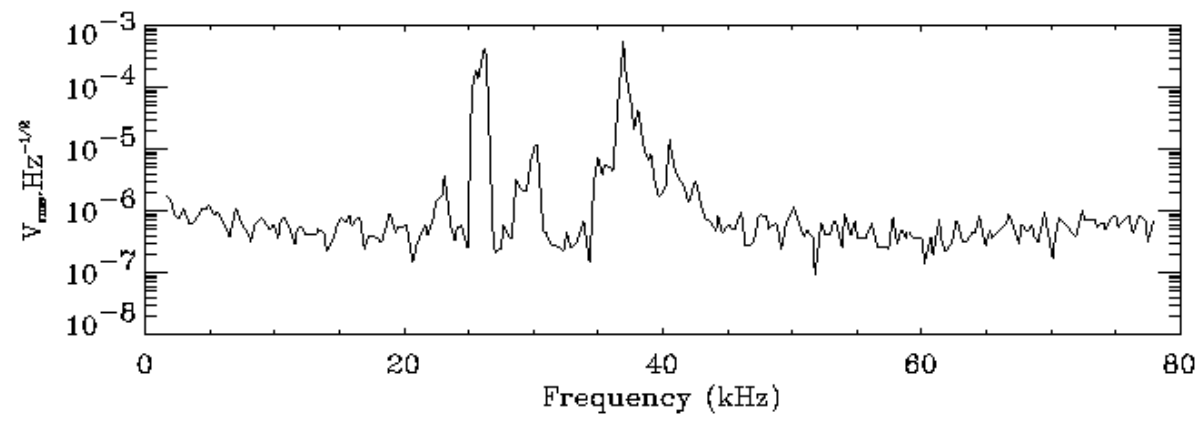

Fig. 11. A single frequency spectrum derived from an active sounding sweep, at 13:29:51 UT, for the event shown in Fig. 10.

quency/time spectrogram obtained using all spectra from the Natural Wave mode, whereas the lower panel displays the spectra obtained in the same time interval from the Sounding mode. A comparison shows that the main peak obtained in the Sounding mode, most likely a true resonance at the electron plasma frequency $F_{p}$, stays at about the same position, close to $40 \mathrm{kHz}$, during the whole interval, whereas the waves observed in the Natural Wave mode behave quite differently.

At the start of the interval, the frequency spectrum of the emission is rather wide. It is primarily concentrated around the electron plasma frequency and higher, such that these well formed oscillations look like a wide, single wave band on the spectrogram. As the plasma frequency is much higher than the electron gyro-frequency in this region, these oscillations can be identified as Langmuir waves. At about 13:22 UT, and later at about 13:32 UT, the spectrum undergoes a significant change: the previously single structure splits into two bands, and at the same time, the overall wave intensity decreases. One of the two wave bands continues to follow the plasma frequency variations, while the central frequency of the other first decreases, and then increases again (probably in conjunction with variations in the shock configuration). At 13:50, the two wave bands merge again. A remarkable feature is that the frequency of the lower frequency mode decreases to frequencies as low as 0.1 of the characteristic frequency of the higher frequency mode. In an attempt to answer the question of whether these oscillations represent eigen-modes of the plasma with a particular distribution of particles (electrons in this case) or if they are forced, we have analysed spectra obtained in the Sounding mode. Many of them display peaks in several frequencies in addition to the $F_{p}$ resonance. In the example of Fig. 11, a wave packet appears close to the plasma frequency at $40 \mathrm{kHz}$, and another appears at about $28 \mathrm{kHz}$. The displayed spectrum is the "active" frequency spectrum from the sounder (see Sect. 2.2). Each bin is compared to the "passive" one, acquired $13 \mathrm{~ms}$ 


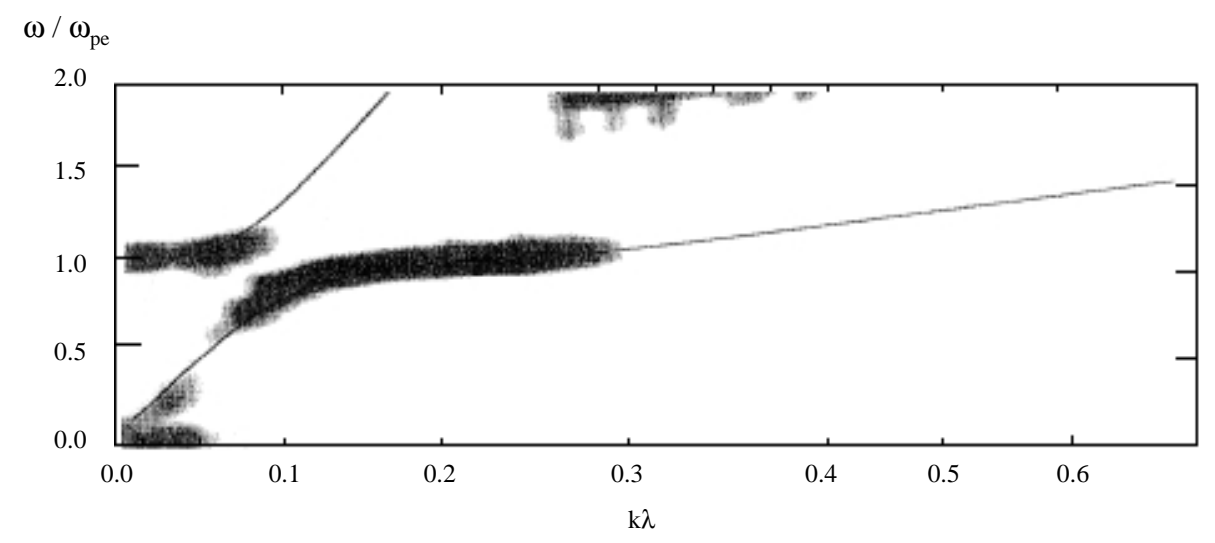

Fig. 12. Wave spectrum from a Vlasov simulation (from Shriver et al., 2000). The real frequency $(\omega)$ is normalized to the total electron plasma frequency $\left(\omega_{p e}\right)$; the wavenumber $(k)$ is multiplied by the cold electron Debye length, $(\lambda)$.

before. Both the $40 \mathrm{kHz}$ and the lower frequency packets indicate for some frequencies a significant "active to passive" ratio, each of which could possibly be a resonance. After analysing several spectra, however, we were convinced that only the peak at $F_{p}$ is a true resonance signature. Other natural wave packets, of very short duration, often shorter than the $13 \mathrm{~ms}$ best possible resolution of the instrument, can suddenly appear in an "active" spectrum, superposed on the specific sounding response. This fact explains the presence of the remnant bursts below $F_{p}$ observed in the "active" spectrogram of Fig. 10. The lower frequency peak on the figure is due to the natural emissions which happened to be rather intense at that moment (13:29:51 UT). In conclusion, the sounder data brings an interesting piece of information to the detailed study of these waves, which is beyond the scope of this paper.

We would like, nevertheless, to notice that their properties resemble the properties of the electron- acoustic waves. This oscillation mode exists when the electron distribution has two constituents, cold and hot. The hot electron population is a typical feature of the solar wind, but its density is quite small. However, in the vicinity of the quasi-perpendicular shock front in an electron foreshock region, there exists an additional population of reflected electrons. Their density and velocity distribution depend upon the shock strength and the angle of the upstream magnetic field with respect to the shock normal (Wu, 1984; Leroy and Mangeney, 1984). These two parameters can vary with time, or along the spacecraft trajectory; hence, a change of the electron-acoustic waves is most effectively generated. The generation of these waves was studied by several authors (Dum, 1990; Cairns, 1989; Sotnikov et al., 1995). Recently, their generation and saturation have been studied via a computer simulation using Particle In Cell and Vlasov 1D codes (Schriver et al., 2000). A result of the simulation is shown in Fig. 12, with a copy of Fig. 8 of the cited paper. This plot illustrates the frequency/ $\boldsymbol{k}$ vector dependence of the triggered oscillations, shows that the wave generation occurs in two frequency bands belonging to two branches of oscillations. The characteristic fre- quency of the waves with the highest growth rate is determined by the electron distribution function, and thus, varies as a function of it. Such behaviour implies that a variable separation of the frequency bands, as seen in Fig. 10, reflects variations in the electron distribution function. Now, if the particle distribution function is determined by the position of the satellite with respect to different regions of the foreshock, then we are in possession of a very valuable tool for remotely studying the bow shock shape and dynamics. Different natural emission features observed at the four positions inside the foreshock, as illustrated from the four views in Fig. 9, could be translated into both the distance and the inclination of the shock at the four different points of connection.

\subsection{The density gradient tool}

Figure 13 shows an example of plasma penetration into the magnetopause boundary layer in two successive surges. Numerous examples of such plasma penetrations have been observed by the Cluster spacecraft. This example has been chosen on the simple ground that it was one of the first orbits during which the four spacecraft were operating simultaneously, in this case, during the interference campaign which closed the commissioning phase. One frequency/time spectrogram is shown in the upper panel, with the four density profiles in the lower panel.

The appearance of this event is not very different from what has been observed with other missions (La Belle et al., 1987). The position of the plasma frequency is marked by a low-frequency cutoff in the natural wave emissions presented in this region, often emphasised by a single line emission of strong intensity. All the density profiles display sharp gradients at the boundary of the structure, but differ in their details. The width of the first structure (12:36:15-12:37:00 UT), for instance, differs from SALSA to TANGO, whereas it is of the same order of magnitude for the second structure (12:38:1512:39:15 UT). Several approaches can be used to study the shape and dynamics of a structure. The estimation of the velocity of a rigid discontinuity has been briefly presented in 


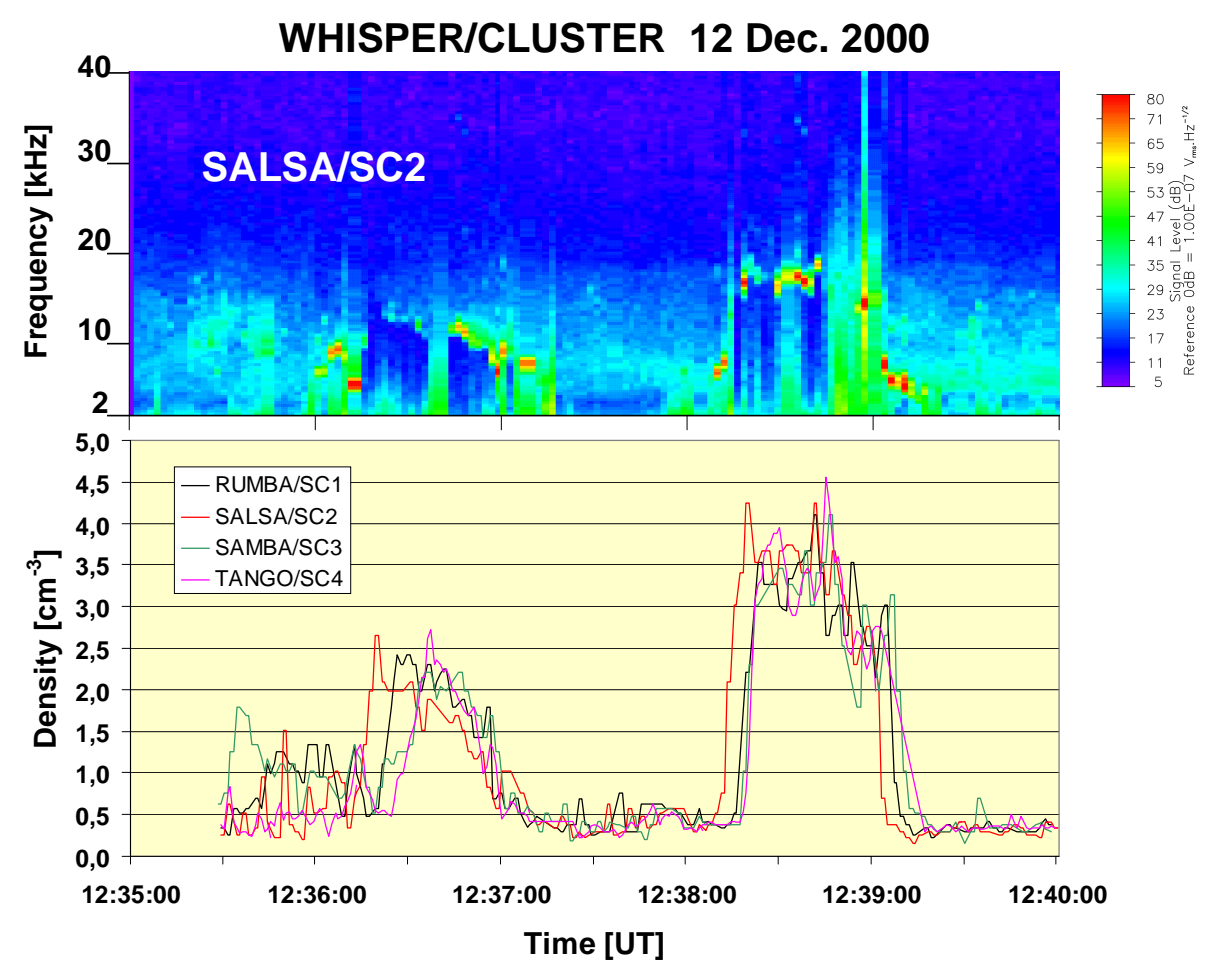

Fig. 13. Two successive plasma transfer events in the magnetopause boundary layer ( $17 \mathrm{MLT}, 28^{\circ}$ latitude, $\left.17.2 R_{E}\right)$. The bottom panel shows the density profiles derived from the four frequency/time spectrograms analogous to the one in the upper panel.

an example in Sect. 3.2. We wish to present now another approach, namely the derivation of the density gradient vector, using data of the event presented in Fig. 13. The concept is simple: four values of a given parameter, here the scalar density, taken at the same time at four known positions, will provide an estimation of the density gradient vector. The estimation will be representative only if the gradient is actually constant inside the tetrahedron volume, a condition that cannot be tested in a simple way. Our aim here is to give a brief summary of the method used in practice, and present results obtained with the gradient tool, rather than discussing its validity in detail.

The least square method of determining the spatial gradient of a parameter using data acquired simultaneously from four (or more) spacecraft has been described by Harvey (1998). It turns out that the spatial gradient can be expressed in terms of the inverse of a symmetric tensor formed from the relative positions of the spacecraft. In the special case of four spacecraft, this tensor is referred to as the volumetric tensor (its determinant is $(3 V / 8)^{2}$, where $V$ is the volume of the tetrahedron). The tensor is

$R_{i j}=\frac{1}{4} \sum_{\alpha=1}^{4}\left(r_{\alpha}^{i}-r_{b}^{i}\right)\left(r_{\alpha}^{j}-r_{b}^{j}\right)$

where $r_{\alpha}^{i}$ and $r_{\alpha}^{j}$ are the three coordinates of the position of the spacecraft $\alpha(\alpha=1,2,3$ or 4$)$, and $r_{b}^{i}$ are the coordinates of the position of the centre of mass of the four spacecraft. The volumetric tensor describes basic geometrical properties of the tetrahedron defined by the four spacecraft. The eigenvalues can be used to define the characteristic size $L$, the elongation $E$ and the planarity $P$, and the eigenvectors define the directions of elongation $e_{E}$ and planarity $e_{P}$ (Robert et al., 1998). In terms of these geometrical parameters, which are included in the Summary Parameters of the Cluster Scientific Data System, the inverse of the volumetric tensor may be expressed as:

$R_{j i}^{-1}=\frac{4}{L^{2}}$

$\left[e_{E_{j}} e_{E i}+\frac{1}{(1-E)^{2}} e_{L j} e_{L j}+\frac{1}{(1-P)^{2}(1-E)^{2}} e_{P j} e_{P i}\right]$,

where $e_{L}$ is the third, mutually orthogonal direction, $e L=$ $e_{P} x e_{E}$. Finally, it can be shown that the least squares estimation (which for four spacecraft, is, in fact, the exact estimation) of the linear density gradient is:

$\frac{\delta n}{\delta r_{i}}=\frac{1}{2} \frac{1}{4^{2}} \sum_{j}\left[\sum_{\alpha=1}^{4} \sum_{\beta=1}^{4}\left(n_{\alpha}-n_{\beta}\right)\left(r_{\alpha}^{j}-r_{\beta}^{j}\right)\right] \times R_{j i}^{-1}$,

where $n_{\alpha}$ is the value of density $n$ measured on the spacecraft $\alpha$.

The density gradient vectors obtained during the crossing of the second structure are shown in Fig. 14. The successive values determined are displayed, projected onto the three planes of the GSE coordinate system. The orbital segment of the centre of mass of the constellation is also projected onto each plane, with a triangle to indicate the point at which 


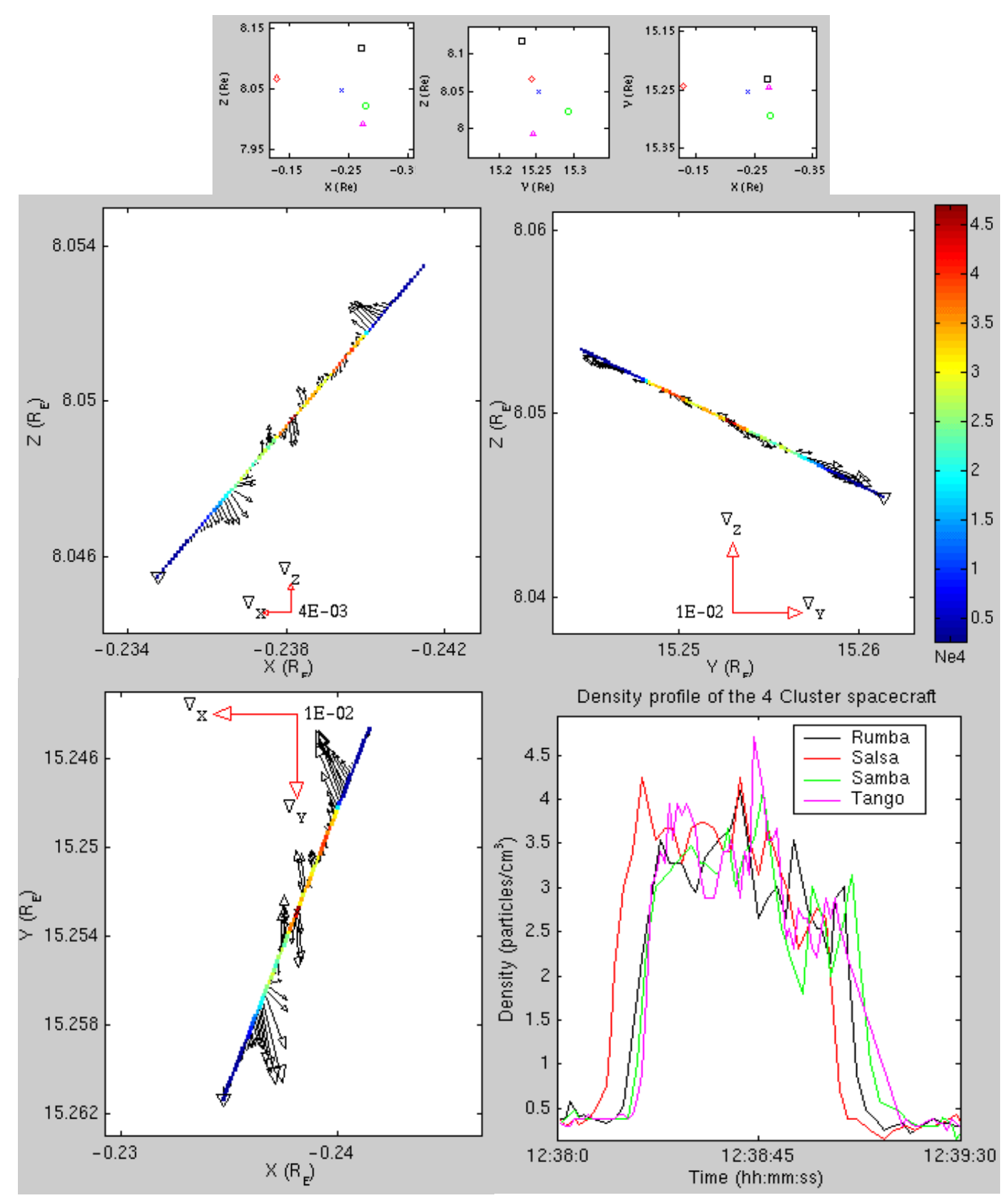

Fig. 14. Results of applying the gradient operator to the second structure of Fig. 13. Top: the positions of the spacecraft and the center of mass (dark blue cross) projected onto the 3 GSE planes. Below: density gradient vectors (part $/ \mathrm{cm}^{3} / \mathrm{km}$ ) marked along a segment of the trajectory and projected onto the 3 GSE planes. The colour of the trajectory indicates the density at the TANGO spacecraft. Bottom right: the four density profiles.

Cluster left the segment. The origins of the successive projected vectors move along the orbital segment as the centre of mass moves. Such a presentation clearly brings additional information (the separation between spacecraft) to the comparison of the density profiles (bottom, right side). Qualitatively, we immediately see the variation in the magnitude and direction of the gradient along the orbital trajectory; the vector remains close to the plane defined by the $x$-axis and the orbital velocity (the vectors are aligned with the orbit in the $z-y$ plane). They are pointing to a low $|x|$ low $y$, high $z$ when entering the structure, and the reverse is true when they are leaving the structure.

Although this result should be interpreted with caution since the "gradient" is an spatially averaged value, we start to separate time and space, and obtain quantitative values. At the particular times when all spacecraft seem to be on the same "slope", as in this case around 12:38:15 UT, the gradient obtained should be truly meaningful. In order to increase the occurrence of the favourable conditions for studying the magnetopause boundary, a tetrahedron size smaller than at 
the start of the mission has to be considered. This situation is foreseen to take place in late 2001 (100 km spacecraft separation, to be compared to $600 \mathrm{~km}$ in December 2000).

\section{Summary and conclusion}

In summary, the first observations from the Whisper instrument on Cluster are startling. The instrument's receiver and wave analyser behave beautifully, and the technology, which was chosen almost twelve years ago, proves the existence of new capabilities, such as the possibility of measuring wave directivity. The sensitivity and the time/frequency resolution are able to show small differences between the four spacecraft, which is the main objective.

Whisper's sounder design proved to be efficient in the vast majority of the cases encountered. The main limitations, which have not yet been fully investigated, are inherent to the technique: the density is derived indirectly, and the resonance recognition process is not $100 \%$ efficient, especially in complex plasmas; and simple instrument limitations exclude some regions or some plasma regimes.

The exploration of regions that are not yet well-known has proven to be fascinating, from plasmapause regions to the solar wind, including the magnetosheath and cusp regions, which sometimes look different from what is described in the text books. The examples shown in this paper are only some of the events which can be studied using Whisper data. WC have now to put together the different pieces of information, to compare instruments, to test the multi-point analysis techniques which have been prepared, and imagine new ones based on real data. A difficult but fascinating challenge is ahead.

Acknowledgements. The authors wish to acknowledge the efforts of a number of people who, at one time or another, worked for the WHISPER experiment: A. Bahnsen, from DSRI, A. Sumner, C. Dunford, J. Thompson (Univ. Sheffield); C. Vasiljevic, E. Guyot, L. Launay, J.-L. Fousset, (LPCE). The WEC, ESA, Dornier, RAL and IABG teams are warmly thanked for their participation and help in the testing and integration of the experiment. Our warm thanks too to engineers at Starsem and ESOC for a faultless launch, and to the JSOC and ESOC teams for supporting complex operations. We are grateful to G. Chanteur (CETP, Vélizy), who derived the value of a discontinuity velocity and associated uncertainties. Several discussions have benefited from the availability of magnetometer data (courtesy of A. Balogh), and we express our thanks to the FGM team. We wish to extend our thanks to those who helped the WHISPER investigators in their contribution to the CSDS, in particular J.P. Thouvenin, H. Poussin, M. Nonon, J.-Y. Prado and J.-C. Kosik of CNES. The WHISPER experiment and software are realised thanks to a CNES contract. The transmitter hardware was designed and built at DSRI.

Topical Editor M. Lester thanks H. Laakso and another referee for their help in evaluating this paper.

\section{References}

Anderson, R. R., Parks, G. K., Eastman, T. E., Gurnett, D. A., and Frank, L. A.: Plasma waves associated with energetic particles streaming into the solar wind from the Earth's bow shock, J. Geophys. Res., 86, 4493-4510, 1981.

Béghin, C. and Kolesnikova, E.: Surface-charge distribution approach for modeling of quasi-static electric antennas in isotropic thermal plasma, Radio Sci., 33, 503-516, 1998.

Cairns, I.: Electrostatic wave generation above and below the plasma frequency by electron beams, Phys. Fluids, B1, 204-213, 1989.

Canu, P., Décréau, P. M. E., Trotignon, J. G., Rauch, J. L., Séran, H. C., Fergeau, P., Lévêque, M., Martin, Ph., Sené, F. X., Le Guirriec, E., Alleyne, H., and Yeraby, K.: Identification of natural plasma emissions with the Cluster - Whisper relaxation sounder, Ann. Geophysicae, this issue, 2001.

Carpenter, D. L., Giles, B. L., Chappell, C. R., Décréau, P. M. E., Anderson, R. R., Persoon, A. M., Smith, A. J., Corcuff, Y., and Canu, P.: Plasmasphere dynamics in the duskside bulge region: a new look at an old topic, J. Geophys. Res., 98, 19, 243-271, 1993.

Chanteur, G.: Spatial interpolation for four spacecraft: Theory, in: Analysis methods for Multi-Spacecraft data, (Eds) Paschmann, G. and Daly, P. W., ISSI scientific Report SR-001, 349-369, 1998.

Décréau, P. M. E., Fergeau, P., Lévêque, M., Martin, Ph., Randriamboarison, O., Sené, F. X., Trotignon, J. G., Canu, P., de Féraudy, H., Bahnsen, A., Jespersen, M., Mögensen, P. B., Iversen, I., Dunford, C., Sumner, A., Woolliscroft, L. J. C., Gustafsson, G., and Gurnett, D. A.: "Whisper", a sounder and High frequency wave analyser experiment, ESA SP-1159, 5167, 1993.

Décréau, P. M. E., Fergeau, P., Krasnoselskikh, V., Lévêque, M., Martin, Ph., Randriamboarison, O., Sené, F. X., Trotignon, J. G., Canu, P., Mögensen, P. B. and Whisper investigators: Whisper, a resonance sounder and wave analyser: performances and perspectives for the Cluster mission, Space Sci. Rev., 79, 93-105, 1997.

Décréau, P. M. E., Béghin, C., and Parrot, M.: Global characteristics of the cold plasma in the equatorial plasmapause region as deduced from the GEOS 1 mutual impedance probe, J. Geophys. Res., 87, 695-712, 1982.

Dubouloz, N., Pottelette, R., Malingre, M., Holmgren, G., and Lindqvist, P. A.: Detailed analysis of broadband electrostatic noise in the dayside auroral zone, J. Geophys. Res., 96, 35653579, 1991.

Dum, C. T.: Simulation of Plasma Waves in the Electron Foreshock: The Generation of Downshifted Oscillations, J. Geophys. Res., 95, 8123-8131, 1990.

Etcheto, J., Christiansen, P. J., Gough, M. P., and Trotignon, J. G.: Terrestrial Continuum radiation observations with GEOS-1 and ISEE-1, Geophys. Res. Let., 9, 1239-1242, 1982.

Etcheto, J., Belmont, G., Canu, P., and Trotignon, J. G.: Active sounder experiments on GEOS and ISEE, ESA SP6195, 39-46, 1983.

Etcheto, J. and Faucheux, M.: Detailed study of electron plasma waves upstream of the Earth's bow shock, J. Geophys. Res., 89, 6631-6653, 1984.

Fuselier, S. A., Gurnett, D. A., and Fitzenreiter, R. J.: The downshift of electron plasma oscillations in the electron foreshock region, J. Geophys. Res., 90, 3935-3946, 1985. 
Gurnett, D. A.: The Earth as a radio source: the nonthermal continuum, J. Geophys.Res. 80, 2751-2763, 1975.

Gustafsson, G., Boström, R., Holback, B., Holmgren, G., Lundgren, A., Stasiewicz, K., Ahlen, L., Mozer, F. S., Pankow, D., Harvey, P., Berg, P., Ulrich, R., Pedersen, A., Schmidt, R., Butler, A., Fransen, A. W. C., Klinge, D., Thomsen, M., Fälthammar, C.-G., Lindqvist, P.-A., Christenson, S., Holtet, J., Lybekk, B., Sten, T. A., Tanskanen, P., Lappalainen, K., and Wygant, J.: The electric filed and wave experiment for the Cluster mission, Space Sci. Rev., 79, 137-156, 1997.

Gustafsson, G., André, M., Carozzi, T., Eriksson, A. I., Fälthammar, C.-G., Grard, R., Holmgren, G., Holtet, J. A., Ivchenko, N., Karlsson, T., Khotyaintsev, Y., Klimov, S., Laakso, H., Lindqvist, P.-A., Lybekk, B., Marklund, G., Mozer, F., Mursula, K., Pedersen, A., Popielawska, B., Savin, S., Stasiewicz, K., Tanskanen, P., Vaivads, A., and Wahlund, J.-E.: First results of electric field and density observations by Cluster EFW based on initial months of observations, Ann. Geophyicae, this issue, 2001.

Harvey, C. C.: Spatial gradient and the volumetric tensor, in: Analysis methods for Multi-Spacecraft data, (Eds) Paschmann, G. and Daly, P. W., ISSI scientific Report SR-001, pp. 307-322, 1998.

Hulqvist, B.: The Viking project, Geophys. Res. Let. 74, 379-382, 1987.

LaBelle, J., Treumann, R. A., Haerendel, G., Bauer, O. H., Paschmann, G., Baumjohan, W., Lühr, H., Anderson, R. R., Koons, H. C., and Holzworth, R. H.: AMPTE-IRM observations of waves associated with flux transfer events in the magnetosphere, J. Geophys. Res., 92, 5827-5843, 1987.

Leroy, M. M. and Mangeney, A.: A theory of energization of solar wind electrons by the Earth's bow shock, Ann. Geophysicae, 2, 440-456, 1984.

Meyer-Vernet, N. and Perche, C.: Tool kit for antennae and thermal noise near the plasma frequency, J. Geophys. Res., 94, 24012415, 1989.

Pedersen, A., Cornilleau-Wherlin, N., De La Porte, B., Roux, A., Bouabdellah, A., Décréau, P. M. E., Lefeuvre, F., Sené, F. X., Gurnett, D., Huff, R., Gustafsson, G., Holmgren, G., Woolliscroft, L., Alleyne, H. St. C., Thompson, J. A., and Davies, P. N.
H.: The Wave Experiment Consortium (WEC), Space Sci. Rev., 79, 157-193, 1997.

Robert, P., Roux, A., Harvey, C. C., Dunlop, M. W., Daly, P. W., and Glassmeier, K.-H.: Tetrahedron Geometric Factors, in: Analysis methods for Multi-Spacecraft data, (Eds) Paschmann, G. and Daly, P. W., ISSI scientific Report SR-001, 323-348, 1998.

Schriver, D., Ashour-Abdalla, M., Sotnikov, V., Hellinger, P., Fiala, V., Bingham, R., and Mangeney, A.: Excitation of electron acoustic waves near the electron plasma frequency and at twice the plasma frequency, J. Geophys. Res., 105, 12, 919-927, 2000.

Sotnikov, V. I., Schriver, D., Ashour-Abdalla, M., Ernstmeyer, J., and Myers, N.: Excitation of electron acoustic waves by a gyrating electron beam, J. Geophys. Res., 100, 19, 765-772, 1995.

Stasiewicz, K.: OVT Visualization Tool-2 for CLUSTER, User Guide, Copyright (C) 2000 by the OVT team, version 2.0, http://ovt.irfu.se, 2001.

Thiel, J. and Debrie, R.: Electrostatic wave potential at the plasma and upper-hybrid resonances, J. Plasma Phys., 25, 239-254, 1981.

Trotignon, J. G., Etcheto, J., and Thouvenin, J. P., Automatic determination of the electron density measured by the relaxation sounder on-board ISEE 1, J. Geophys. Res. 91, 4302-4320, 1986.

Trotignon J. G., Décréau, P. M. E., Rauch, J. L., Randriamboarison, O., Krasnoselskikh, V., Canu, P., Alleyne, H., Yeraby, K., Le Guirriec, E., Séran, H. C., Sené, F. X., Martin, Ph., Lévêque, M., and Fergeau, P.: How to determine the thermal electron density and the magnetic field strength from the CLUSTER/WHISPER observations around the Earth, Ann. Geophysicae, this issue, 2001.

Woolliscroft, L. J. C., Alleyne, H. ST. C., Dunford, C. M., Sumner, A., Thompson, J. A., Walker, S. N., Yearby, K. H., Buckley, A., Chapman, S., Gough, P., and the DWP investigators: The Digital Wave-Processing experiment on Cluster, Space Sci. Rev., 79, 209-231, 1997.

Wu, C. S.: A fast Fermi process: energetic electrons accelerated by a nearly perpendicular Bow Shock, J. Geophys. Res., 89, 8857$8862,1984$. 